\title{
An Air Jet Distortion Generation System
}

\author{
M. Sivapragasam, ${ }^{1}$ S. Ramamurthy, ${ }^{2}$ M. D. Deshpande, ${ }^{1}$ and P. White ${ }^{3}$ \\ ${ }^{1}$ Department of Automotive and Aeronautical Engineering, Faculty of Engineering and Technology, \\ M. S. Ramaiah University of Applied Sciences, Bangalore 560 058, India \\ ${ }^{2}$ National Civil Aircraft Development Programme, CSIR-National Aerospace Laboratories, Bangalore 560 017, India \\ ${ }^{3}$ Faculty of Engineering and Computing, Coventry University, Coventry CV1 5FB, UK
}

Correspondence should be addressed to M. Sivapragasam; sivapragasam.aae.et@msruas.ac.in

Received 22 May 2014; Revised 4 September 2014; Accepted 7 September 2014; Published 23 October 2014

Academic Editor: Ningsheng Feng

Copyright (c) 2014 M. Sivapragasam et al. This is an open access article distributed under the Creative Commons Attribution License, which permits unrestricted use, distribution, and reproduction in any medium, provided the original work is properly cited.

\begin{abstract}
An air jet distortion generation system is developed to simulate the distorted flow field ahead of gas turbine engines in ground test facility. The flow field of a system of four jets arranged circumferentially and issuing into a confined counterflow was studied experimentally and numerically. The total pressure distortion parameters were evaluated at the Aerodynamic Interface Plane (AIP) for several values of mass flow ratios. Since the total pressure loss distribution at the AIP is characteristically "V" shaped, the number of jets was increased to obtain total pressure distributions as required for gas turbine engine testing. With this understanding, a methodology has been developed to generate a target total pressure distortion pattern at the AIP. Turbulent flow computations are used to iteratively progress towards the target distribution. This methodology was demonstrated for a distortion flow pattern typical of use in gas turbine engine testing using twenty jets, which is a smaller number than reported in the literature. The procedure converges with a root-mean-square error of $3.836 \%$ and is able to reproduce the target pattern and other distortion parameters.
\end{abstract}

\section{Introduction}

The performance of a modern fighter aircraft is heavily influenced by the performance of its propulsion system. These aircraft perform high angle of attack and high angle of sideslip maneuvers, which puts severe demand on the gas turbine engine. The engine compression system is particularly vulnerable to nonuniform or distorted flow in the inlet duct. The compressors are designed for uniform inlet flows and suffer from performance degradation due to nonuniform inflow conditions leading the compressor to aerodynamic instabilities like rotating stall and surge. The distortion in the inlet flow field can be in static or total pressures or temperatures or velocities. The total pressure distortion is the most common type and also has the most deleterious effect on the performance of the compression system. The total pressure distortion can be either in circumferential or radial directions and such total pressure patterns are called "classical" distortion patterns. The total pressure distortion profiles such as those occurring at flight conditions are termed "complex" (or "composite") distortion patterns and can have both circumferential and radial nonuniformities.

The general subject of inlet flow field distortion and its adverse effects on the performance and stability of the compression systems were reviewed by Longley and Greitzer [1] and Sivapragasam and Ramamurthy [2]. The distorted flow field ahead of the compressor is simulated in ground test facilities by various methods. Several such techniques were examined by Beale et al. [3]. Of the various methods of distortion simulation in ground test facilities the distortion screen and the air jet distortion system are deemed most satisfactory.

The distortion screens have been commonly employed for simulating total pressure distorted inlet flow field in test facilities. The screens are simply wire meshes of various porosities secured to a frame and placed ahead of the engine/compressor. The screen porosities are chosen to produce the required pressure drop. The distortion screens are the most preferred method of distortion simulation by virtue of their simplicity. The distortion screens have a 
few disadvantages though, arising mainly out of operational issues. Firstly, a screen can produce only a distinct distortion pattern. Since the engine operability evaluation consists of a number of distortion patterns to be simulated, each pattern simulation requires a different screen. This necessitates the need to interrupt the test process, uninstall the test hardware, and change the screen every time another distortion pattern is to be simulated. The time and cost overruns associated with these interruptions are very high. Secondly, the screens are effective only over a narrow flow range below which they are ineffective and beyond which the holes in the screen choke. Moreover, the distortion screens essentially produce steady-state distortion pattern. However, if dynamic distortion conditions are to be simulated, the screens are incapable of doing this. These considerations have led to the development of other means of inlet distortion simulation.

The air jet distortion system can alleviate many of the operational disadvantages encountered with the conventional distortion screens. The system consists of a number of air jets arranged in a circumferential array and issuing opposite to the primary air flow entering the engine. The jets interact with the primary stream and cause a local total pressure loss due to momentum exchange. The individual mass flow rates from the jets can be varied to obtain a required total pressure pattern ahead of the compressor. Such air jet distortion systems were developed at the NASA Glenn Research Center [4] and at the Arnold Engineering Development Center [5], and more recently by Naseri et al. [6].

The development of such an air jet distortion system, which is the focus of the present study, would require a systematic study and an understanding of the of total pressure loss caused by jets issuing into a counterflow primary stream. A system of four jets issuing into a confined counterflow was studied experimentally and numerically. From this understanding a methodology has been developed to generate a given complex total pressure distortion pattern. The method is based on the computational procedure for multiple jets in counterflow and the quasi-one-dimensional inviscid analysis is used as the starting point to estimate the overall total pressure loss. The methodology thus developed is demonstrated to generate total pressure patterns typical of use in aircraft gas turbine engine testing.

\section{Description of the Flow Field}

A schematic illustration of the flow field under consideration is depicted in Figure 1. Four steady, compressible, turbulent jets of uniform velocity $u_{j}$ and density $\rho_{j}$ issue from a nozzle, each of internal diameter $d_{j}$ into a steady, turbulent, uniform counter stream of velocity $u_{0}\left(u_{j}>u_{0}\right)$, density $\rho_{0}$, and confined within a duct of diameter $D_{0}$. In the present investigation $D_{0}=102 \mathrm{~mm}$ and $d_{j}=7.5 \mathrm{~mm}$. The mass flow of the counterflow stream is $m_{1}=(\pi / 4) D_{0}{ }^{2} \rho_{0} u_{0}$ and that at the jet exit is $m_{2, k}=(\pi / 4) d_{j}^{2} \rho_{j k} u_{j k}$. The total mass flow rate from the jets is

$$
m_{2}=\sum_{k=1}^{N} m_{2, k}
$$

where $N=4$ in the present case. The mass flow rates in the individual jets, $m_{2, k}$, can be varied independently, and they can be either equal or unequal.

The mass flow ratio is defined as the ratio of the jet mass flow rate to the total mass flow rate, $m_{2} / m_{4}$, where $m_{4}=$ $\left(m_{1}+m_{2}\right)$. The station numbering is also shown in Figure 1 . The counterflow stream (far ahead of the jets) is numbered 1 , the jet exit plane 2 , a plane one-duct diameter downstream of the jet exit where the total pressure distortion is evaluated is 3 and the (far downstream, combined jet and counterflow stream) outlet is 4 . In this study station 3 is considered to be the Aerodynamic Interface Plane $(A I P)$. This is the station used to define the total pressure distortion between the inlet and engine [7].

\section{Computational Procedure}

The governing equations for mass and momentum conservation for a turbulent flow field namely, the Reynolds-averaged Navier-Stokes equations are listed below:

$$
\begin{gathered}
\frac{\partial \rho}{\partial t}+\frac{\partial}{\partial x_{i}}\left(\rho u_{i}\right)=0 \\
\frac{\partial}{\partial t}\left(\rho u_{i}\right)+\frac{\partial}{\partial x_{j}}\left(\rho u_{i} u_{j}\right) \\
=\frac{\partial p}{\partial x_{i}}+\frac{\partial}{\partial x_{j}}\left[\mu\left(\frac{\partial u_{i}}{\partial x_{j}}+\frac{\partial u_{j}}{\partial x_{i}}-\frac{2}{3} \delta_{i j} \frac{\partial u_{l}}{\partial x_{l}}\right)\right] \\
+\frac{\partial}{\partial x_{j}}\left(-\rho \overline{u_{i}^{\prime} u_{j}^{\prime}}\right), \\
\frac{\partial}{\partial t}(\rho E)+\nabla \cdot[\vec{v}(\rho E+p)] \\
=\nabla \cdot\left[k_{\text {eff }} \nabla T+\left(\overline{\bar{\tau}}_{\text {eff }} \cdot \vec{v}\right)\right]+S_{h},
\end{gathered}
$$

where $u_{i}$ and $u_{i}^{\prime}$ are the mean and fluctuating velocity components, $\rho$ is the fluid density, $\mu$ is the viscosity, $p$ is the pressure and $k_{\text {eff }}$ is the effective thermal conductivity. The Reynolds stresses are related to the mean velocity gradients by

$$
-\rho \overline{u_{i}^{\prime} u_{j}^{\prime}}=\mu_{t}\left(\frac{\partial u_{i}}{\partial x_{j}}+\frac{\partial u_{j}}{\partial x_{i}}\right)-\frac{2}{3}\left(\rho k+\mu_{t} \frac{\partial u_{i}}{\partial x_{i}}\right) \delta_{i j},
$$

where $\mu_{t}$ is the turbulent viscosity and $\delta_{i j}$ is the Kronecker delta function. Two additional transport equations, one for the turbulence kinetic energy, $k$, and another for its dissipation rate, $\varepsilon$, are also solved using the standard $k-\varepsilon$ turbulence model, and the model equations are

$$
\begin{aligned}
& \frac{\partial}{\partial t}(\rho k)+\frac{\partial}{\partial x_{i}}\left(\rho k u_{i}\right) \\
& \quad=\frac{\partial}{\partial x_{j}}\left[\left(\mu+\frac{\mu_{t}}{\sigma_{k}}\right) \frac{\partial k}{\partial x_{j}}\right]+G_{k}+G_{b}-\rho \varepsilon-Y_{M}+S_{k}
\end{aligned}
$$




$$
\begin{aligned}
\frac{\partial}{\partial t}(\rho \varepsilon) & +\frac{\partial}{\partial x_{i}}\left(\rho \varepsilon u_{i}\right) \\
= & \frac{\partial}{\partial x_{j}}\left[\left(\mu+\frac{\mu_{t}}{\sigma_{\varepsilon}}\right) \frac{\partial \varepsilon}{\partial x_{j}}\right]+C_{1 \varepsilon} \frac{\varepsilon}{k}\left(G_{k}+C_{3 \varepsilon} G_{b}\right) \\
& -C_{2 \varepsilon} \rho \frac{\varepsilon^{2}}{k}+S_{\varepsilon} .
\end{aligned}
$$

The turbulence viscosity is calculated in this model as

$$
v_{t}=C_{\mu} \frac{k^{2}}{\varepsilon},
$$

where $C_{\mu}$ is a constant. The model constants are assigned the following values [8]:

$$
\begin{gathered}
C_{1 \varepsilon}=1.44, \quad C_{2 \varepsilon}=1.92, \quad C_{\mu}=0.09, \\
\sigma_{k}=1.0, \quad \sigma_{\varepsilon}=1.3 .
\end{gathered}
$$

The governing equations are solved numerically using the commercial finite-volume method based code ANSYS FLUENT. The time-averaged computations were performed for jet-to-counter-flow mass flow ratios $\left(m_{2} / m_{4}\right)$ ranging from 0.055 to 0.197 for equal mass flow rates in the jets and from 0.190 to 0.352 for varying mass flow rates in the four jets. The counterflow stream inlet and the combined flow outlet were placed at $4.9 D_{0}$ and $-4.9 D_{0}$, respectively, from the jet exit.

The total and static pressure boundary conditions were specified at the counterflow inlet and the jet exit. The pressure boundary conditions at the jet exit corresponded to sonic jet exit velocity. In the air jet distortion system of NASA [4] also the jets were choked. In all the studies reported in this paper the jets remained choked. The exit static pressure was specified at the combined outlet boundary. The total temperature was specified at the counterflow inlet and the jet exits. On the main duct walls and on the walls of the jet stem the no-slip boundary condition was imposed.

The turbulence intensity at the counterflow stream inlet and jet exit were $0.3 \%$ and $2.0 \%$, respectively, based on the mean axial velocity at these locations. They were obtained from measurements by a hot-wire anemometer and were imposed as turbulence boundary conditions for the computations. The pressure-velocity coupling was achieved by the SIMPLE algorithm [9]. All calculations were carried out in double-precision arithmetic.

The computational grid consisted of 962,189 cells. A grid independence study was performed earlier with a single jet (of internal diameter $d_{j}=10 \mathrm{~mm}$ ) located at the axis of the counterflow duct using coarse, medium and fine grid systems. The Grid Convergence Index [10] was calculated and the numerical uncertainty in the calculation of total pressure loss $\lambda_{p 0}$ (see (7)) in terms of the discretization error was $0.90 \%$ and $0.57 \%$, and in the calculation of distortion index (DI) (see (8)) it was $0.96 \%$ and $0.85 \%$ for the medium and fine grids, respectively. Since the medium grid had a reasonably low value of discretization error it was chosen. The computational grid for the four jets case, and later for twenty jets, had relative grid spacing similar to that employed for the single jet medium grid case.

\section{Experimental Procedure}

An experimental facility was designed and built to investigate the characteristics of the counterflow jets and this facility is shown in Figure 2. The counterflow air stream is supplied by a centrifugal blower, passing through the settling chamber which consists of honeycomb and turbulence reduction screens and a contraction and then entering the test section. The counterflow mass flow rate can be controlled by a conical throttle at the exit of the straight duct which can be moved axially by means of a lead screw and a nut. The counterflow stream mass flow rate was calculated by measuring the total and wall static pressures and total temperature at a location $2.7 D_{0}$ upstream of the jet injector.

The high-pressure air for the jets was sourced from a central Compressed Air Facility controlled through a manual valve. The four jets were supplied with high pressure air through individual supply lines controlled by individual control valves. The mass flow rates through the jets were calculated by measuring the wall static pressure in the jet supply lines. The wall static pressure was calibrated against the mass flow rate using a Micromotion Coriolis type mass flow meter; the four supply lines for the jets were individually calibrated. The static pressure in the jet supply lines was measured by a Statham model PA-208TC pressure transducer in conjunction with a Scani-Valve model 48J4-1052. By adjusting the counterflow and the jets' mass flow rates the mass flow ratio $m_{2} / m_{4}$ was varied.

4.1. Measurement of Total Pressure Distortion. The total pressure loss due to the jets in counterflow arrangement and the total pressure pattern was obtained from total pressure measurements made at five axial locations behind the jet injection stem. The total pressure measurements were made by forty total pressure probes. These probes were arranged in eight equiangularly spaced rakes, each rake consisting of five probes located at the centres of equal annular ring areas of the main duct. The location of the probes is shown in Figure 3 and is in accordance with AIR 1419 [11]. The total pressure rakes can be installed at one of the five axial locations downstream of the jet injection.

The pressures were measured by ESP-32HD miniature electronic differential pressure scanners. The signals from the transducers were acquired by an Agilent 34970A Data Acquisition/Switch Unit through RS-232 interface and an on-line data acquisition program was written in National Instruments LabVIEW to acquire and process the experimental data. The uncertainties in the experimental data were estimated by the method of Kline and McClintock (see, Holman [12]). The uncertainty in $m_{2} / m_{4}$ is estimated to be about $0.5 \%$ and that in $\lambda_{p 0}$ and $D I$ are $0.6 \%$ and $1.2 \%$, respectively. 

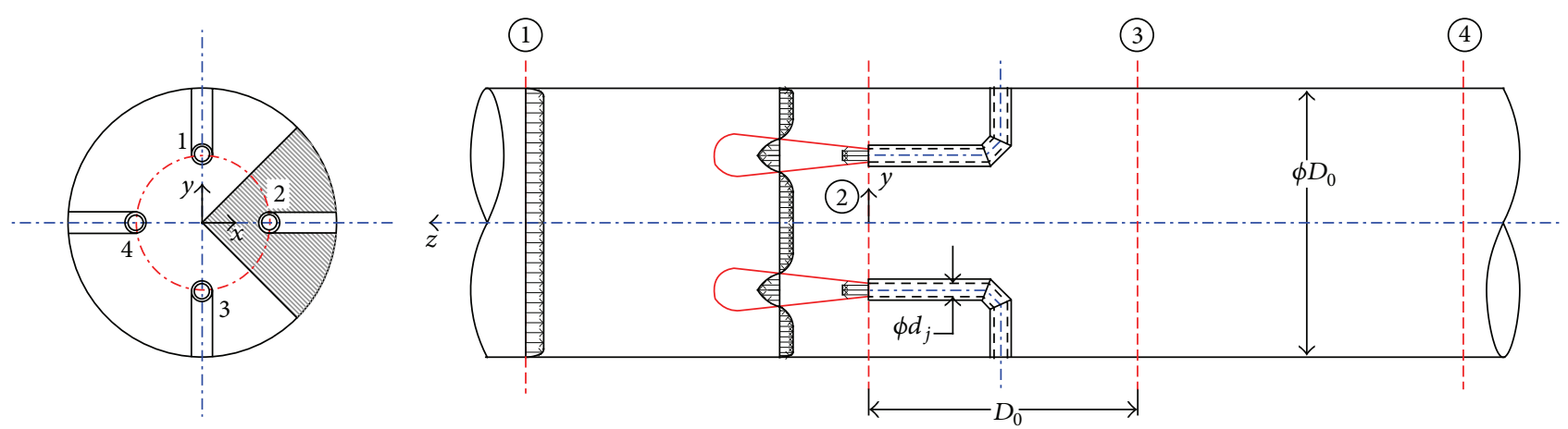

FIGURE 1: Schematic description of the flow field of four circumferentially arranged jets in confined counterflow.

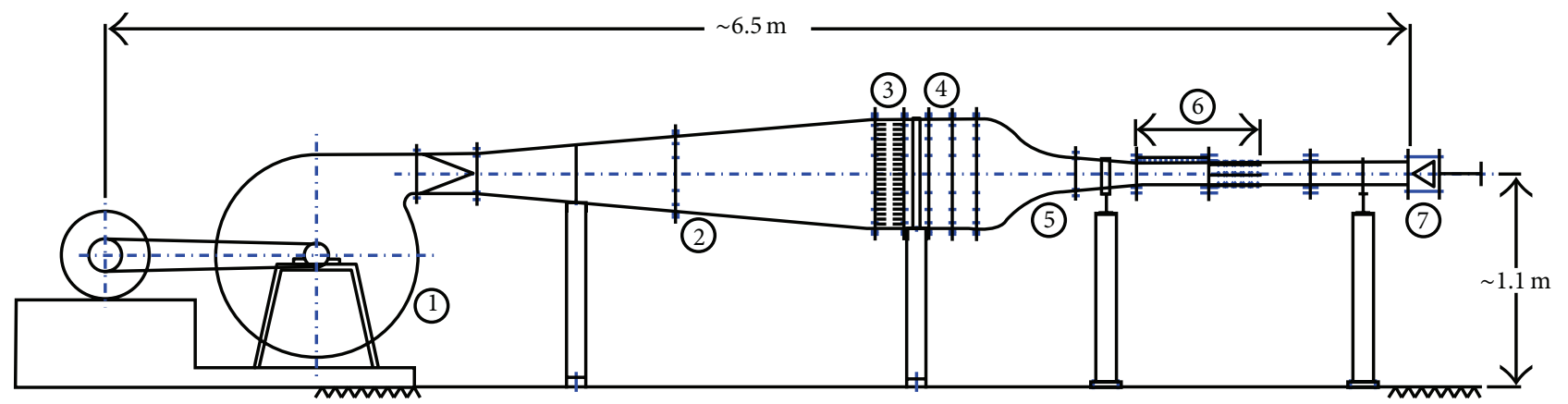

Figure 2: Layout of the test facility; the major components are numbered and are (1) centrifugal blower, (2) diffuser, (3) honeycomb, (4) screens, (5) contraction, (6) test section, and (7) exit throttle.

\section{Procedure for Fixing Mass Flow Rates in the Jets for the Four-Jet System}

The potential capability of the air jet distortion system in producing non-uniform total pressure patterns can greatly be tapped by injecting unequal mass flow rates in the jets. For a given number of jets in the system the possible permutations of mass flow rates amongst the jets are very large. A strategy is to be adopted to keep the mass flow rate combinations to a realizable level. Let a (large) value of mass flow rate in one of the jets be $100 \%$. Then the mass flow rates in the other jets can be fractions of the mass flow rate in one jet, say, $100 \%$, $75 \%, 50 \%$ and $25 \%$, respectively. Thus for a four jet system with each one jet being set at any one of the four settings $(100 \%, 75 \%, 50 \%$ and $25 \%)$ the total number of permutations is $4^{4}=256$. The four settings chosen are arbitrary and the intermediate values and zero are not included since the purpose here is to establish a procedure. Even with only four jets, each with four settings, this is a very large number to perform parametric studies. However, by a careful look at the physical arrangement of the four jets and the permutations of mass flow rates in the four jets one can eliminate those which can be obtained by rotation and mirror symmetry. For example, if the mass flow rates in the four jets are arranged as $\{100 \%: 75 \%: 50 \%: 25 \%\}$, this permutation is the same as $\{75 \%: 50 \%: 25 \%: 100 \%\},\{50 \%: 25 \%: 100 \%: 75 \%\}$ and $\{25 \%: 100 \%: 75 \%: 50 \%\}$ due to same cyclic order. Also, consider the combination $\{100 \%: 75 \%: 50 \%: 25 \%\}$ which is the same set as $\{100 \%: 25 \%$ : $50 \%: 75 \%\}$ or
$\{50 \%: 75 \%: 100 \%: 25 \%\}$ due to mirror symmetry. Thus, it is found that only 55 sets out of these 256 sets are unique.

Out of the 55 unique sets of permutations it is easy to see that there are only 13 combinations of total flow rates from 100 to 400 . The total mass flow rate in this set has the lowest value of 100 (all four jets having 25\% flow rate) and the highest value of 400 (all four jets having 100\% flow rate). From the 55 permutations of mass flow rates, 21 sets were chosen for computations. These 21 sets are chosen to represent the 55 sets which are the only unique sets from the 256 permutations. The selected 21 cases and their mass flow ratios are listed in Table 1. It may be mentioned that the cases 1, 7, 16 and 21 listed in this table have equal mass flow rates in the four jets.

\section{Results and Discussion}

The overall total pressure loss and the total pressure distortion characteristics at the AIP are discussed in this section.

6.1. Total Pressure Loss. The loss in total pressure due to the jets mixing with the counterflow stream was quantified by a non-dimensional total pressure loss parameter, which is defined as

$$
\lambda_{p 0}=1-\frac{p_{0, z 2}}{p_{0, z 1}}
$$

where $p_{0, z 1}$ and $p_{0, z 2}$ are the average total pressures at $z / D_{0}=$ 2.69 and $z / D_{0}=-2.47$, respectively. The inlet total pressure 
TABLE 1: Flow cases chosen for computations.

\begin{tabular}{|c|c|c|c|c|c|c|}
\hline \multirow{2}{*}{ Sl. number } & \multirow{2}{*}{$\begin{array}{l}\text { Inlet Mach } \\
\text { number, } M_{1}\end{array}$} & \multicolumn{4}{|c|}{ Jet mass flow rate, $m_{2 j} \%$} & \multirow{2}{*}{$\begin{array}{l}\text { Mass flow } \\
\text { ratio, } m_{2} / m_{4}\end{array}$} \\
\hline & & Jet 1 & Jet 2 & Jet 3 & Jet 4 & \\
\hline 1 & 0.175 & 25 & 25 & 25 & 25 & 0.119 \\
\hline 2 & 0.186 & 50 & 25 & 25 & 25 & 0.137 \\
\hline 3 & 0.214 & 75 & 25 & 25 & 25 & 0.143 \\
\hline 4 & 0.257 & 100 & 25 & 25 & 25 & 0.141 \\
\hline 5 & 0.184 & 50 & 50 & 50 & 25 & 0.184 \\
\hline 6 & 0.248 & 100 & 25 & 50 & 25 & 0.163 \\
\hline 7 & 0.178 & 50 & 50 & 50 & 50 & 0.210 \\
\hline 8 & 0.244 & 100 & 25 & 75 & 25 & 0.182 \\
\hline 9 & 0.186 & 75 & 50 & 50 & 50 & 0.223 \\
\hline 10 & 0.220 & 100 & 75 & 50 & 25 & 0.214 \\
\hline 11 & 0.253 & 100 & 25 & 100 & 25 & 0.193 \\
\hline 12 & 0.188 & 75 & 75 & 50 & 50 & 0.240 \\
\hline 13 & 0.231 & 100 & 50 & 100 & 25 & 0.222 \\
\hline 14 & 0.185 & 75 & 75 & 75 & 50 & 0.261 \\
\hline 15 & 0.217 & 100 & 75 & 100 & 25 & 0.249 \\
\hline 16 & 0.178 & 75 & 75 & 75 & 75 & 0.286 \\
\hline 17 & 0.212 & 100 & 100 & 100 & 25 & 0.268 \\
\hline 18 & 0.183 & 100 & 75 & 75 & 75 & 0.296 \\
\hline 19 & 0.198 & 100 & 100 & 100 & 50 & 0.297 \\
\hline 20 & 0.182 & 100 & 100 & 100 & 75 & 0.329 \\
\hline 21 & 0.175 & 100 & 100 & 100 & 100 & 0.352 \\
\hline
\end{tabular}

was measured in the experimental facility by a Pitot probe located at the centre of the inlet duct at $z / D_{0}=2.69$ and the exit total pressure was calculated as the average total pressure of the forty measurements made with the total pressure rakes at $z / D_{0}=-2.47$.

The total pressure loss evaluated numerically and obtained from experiments is shown in Figure 4. Also shown in this figure is the total pressure loss estimated from a quasi-one-dimensional inviscid analysis of the flow system solving the steady-state continuity, momentum, and energy equations. In the inviscid analysis with four jets, the total mass flow rate from the jets $m_{2}$ was used to estimate the total pressure loss. It can be seen that the computational and experimental results agree well. The numerical and experimental results indicate a nonzero total pressure loss when the jet mass flow rate is zero because of the presence of the jet injectors protruding into the duct.

6.2. Total Pressure Distortion. The total pressure nonuniformity can be quantified by means of a parameter Distortion Index DI, which is defined as, (see, e.g., Seddon and Goldsmith [13])

$$
D I=\frac{p_{0, \text { max }}-p_{0, \text { min }}}{p_{0, \text { ave }}},
$$

where $p_{0, \max }$ and $p_{0, \min }$ are the maximum and minimum total pressures, respectively, and $p_{0 \text {,ave }}$ is the average total pressure, in any plane of interest. The total pressures were measured by forty total pressure probes, arranged circumferentially in eight rakes each having five probes (see Figure 3). The distortion indices were calculated from the forty total pressure measurements from the experiments and similarly from the computations. The distortion indices obtained at several locations downstream of the jet injector are shown in Figure 5. The computational results agree reasonably well with the experimental data. In general, the distortion index has high values for large mass flow ratios. The highest distortion index occurs at a plane just downstream of the jet injector for all mass flow ratios. At far downstream locations low values of distortion index are observed. The values of distortion are higher when unequal mass flow rates are injected from the jets.

6.3. Total Pressure Distribution and Distortion Parameters at the Aerodynamic Interface Plane. The total pressure distribution and distortion parameters at the AIP are examined in this section for a representative flow case whose $m_{2} / m_{4}=0.214$. To quantify total pressure distortion at the AIP (located at $\left.z / D_{0}=-1\right)$ two more distortion descriptors are useful in addition to the Distortion Index (DI) already defined in (8). This is necessary because though the $D I$ is a gross quantity indicating the non-uniformity and is a useful descriptor for comparative purposes [13], it does not distinguish between circumferential and radial distortion components and also the extent of the distorted region cannot be inferred. 


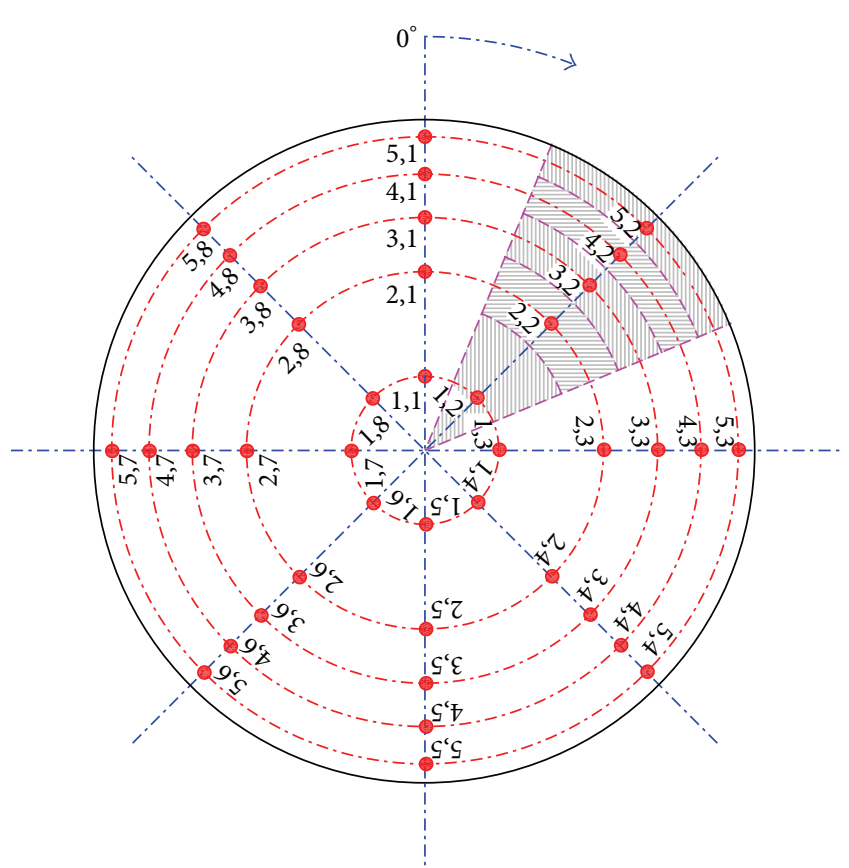

FIGURE 3: Locations where total pressures are measured behind the jet injector. This arrangement is in accordance with AIR1419 (1999).

The circumferential and radial total pressure distortion are defined as (Hubble and Smith [14]; see also Hercock and Williams [15] for a general description of most distortion parameters in common use)

$$
\begin{gathered}
I D C=\frac{p_{0, \text { ring,ave }}-p_{0, \text { ring,min }}}{p_{0, \text { face, ave }}}, \\
I D R=\frac{p_{0, \text { face,ave }}-p_{0, \text { ring,ave }}}{p_{0, \text { face, ave }}},
\end{gathered}
$$

respectively, where $p_{0 \text {,face, ave }}$ is the average total pressure in the plane and $p_{0 \text {,ring,ave }}$ and $p_{0 \text {,ring,min }}$ are the average and minimum total pressures in a ring, respectively. It may be noted that there are as many values of $I D C$ and $I D R$ as the number of rings. IDC is always positive with a larger value indicating a larger loss and lower total pressure. IDR values for all rings together sum to zero.

The total pressure loss contours represented by [1 $\left.\left(p_{0} / p_{01}\right)\right]$, the ring-wise total pressure loss distribution and the circumferential and radial distortion parameters are plotted in Figure 6. In the total pressure loss contours the wake from the jet injectors can be clearly seen. The total pressure loss is high behind the jet struts and low values of losses are found in regions between the wakes of the struts. The pressure distribution is uniform in the innermost ring (ring 1); this is due to the geometry of the flow system as the jet stems do not extend till and beyond ring 1. IDC is found to be highest in ring 4 and the highest IDR is found in ring 1 .

The contours of Mach number and the Mach number distribution at the AIP are also plotted in Figure 6. The high Mach number regions between the wakes of the jet are clearly due to the effect of the confining duct which constrains

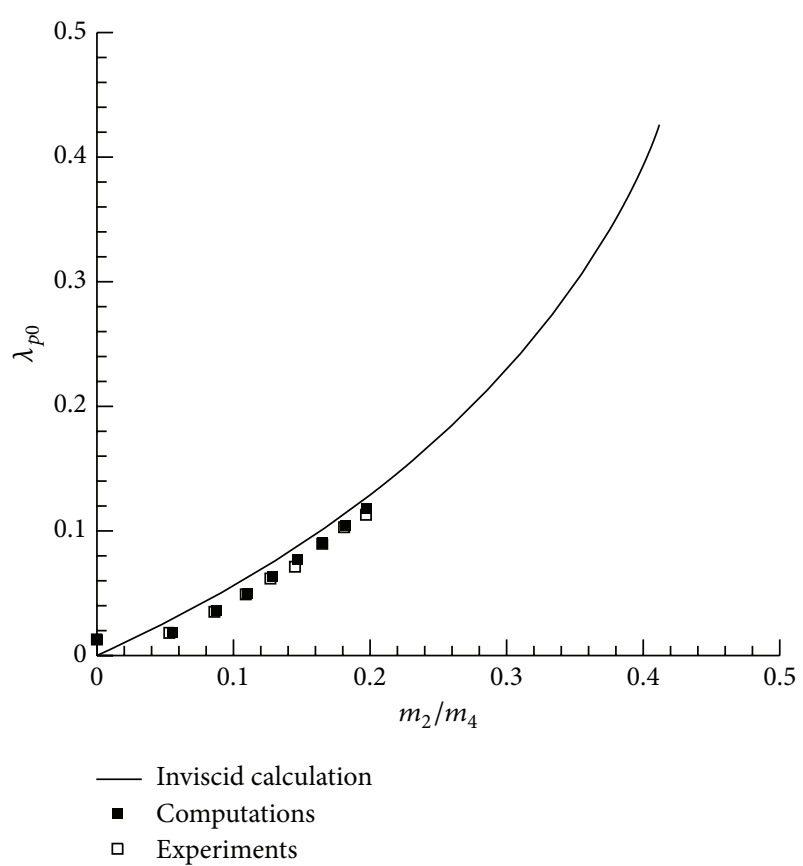

Figure 4: Total pressure loss, $\lambda_{p 0}$, plotted as a function of $m_{2} / m_{4}$; $M_{1}=0.2$.

the radial expansion of the flow. This observation is important because the downstream circumferential total pressure (or Mach number) distribution is intrinsically connected to the number of jets and their arrangement and has a bearing on the design of an air jet distortion system. This statement is further elaborated below.

From the circumferential total pressure loss distributions at the $A I P$ we observe that the total pressure distribution is uniform in the innermost ring (ring 1). For the other rings the peak values occur in between the wakes and the dips are behind the jet injectors. This " $V$ " shaped distribution is characteristic of the four jet system. Of course, the amplitude of the dips and peaks can be varied by varying the mass flow rates in the jets; however, the "V" shaped distribution is not altered. It thus becomes clear that to obtain total pressure distributions as required for gas turbine testing a four jet system would not suffice. The number of jets has to be increased and these issues are discussed in the later sections of this paper.

The study discussed above aids in examining the types of total pressure distribution that can be generated at the AIP. Further it also helps how to invert the problem, that is, for a given target total pressure distribution at the AIP how to select a jet mass flow rate permutation that can achieve the closest desirable total pressure distribution. In Section 8 it will be shown how this idea can be utilized to generate a complex total pressure distortion pattern within the specified constraints and further how its accuracy can be improved by increasing the number of jets and allowing a continuous variation of mass flow rate in each jet.

With the successful modeling of the flow field of four jets in confined counterflow we are now in a position to 

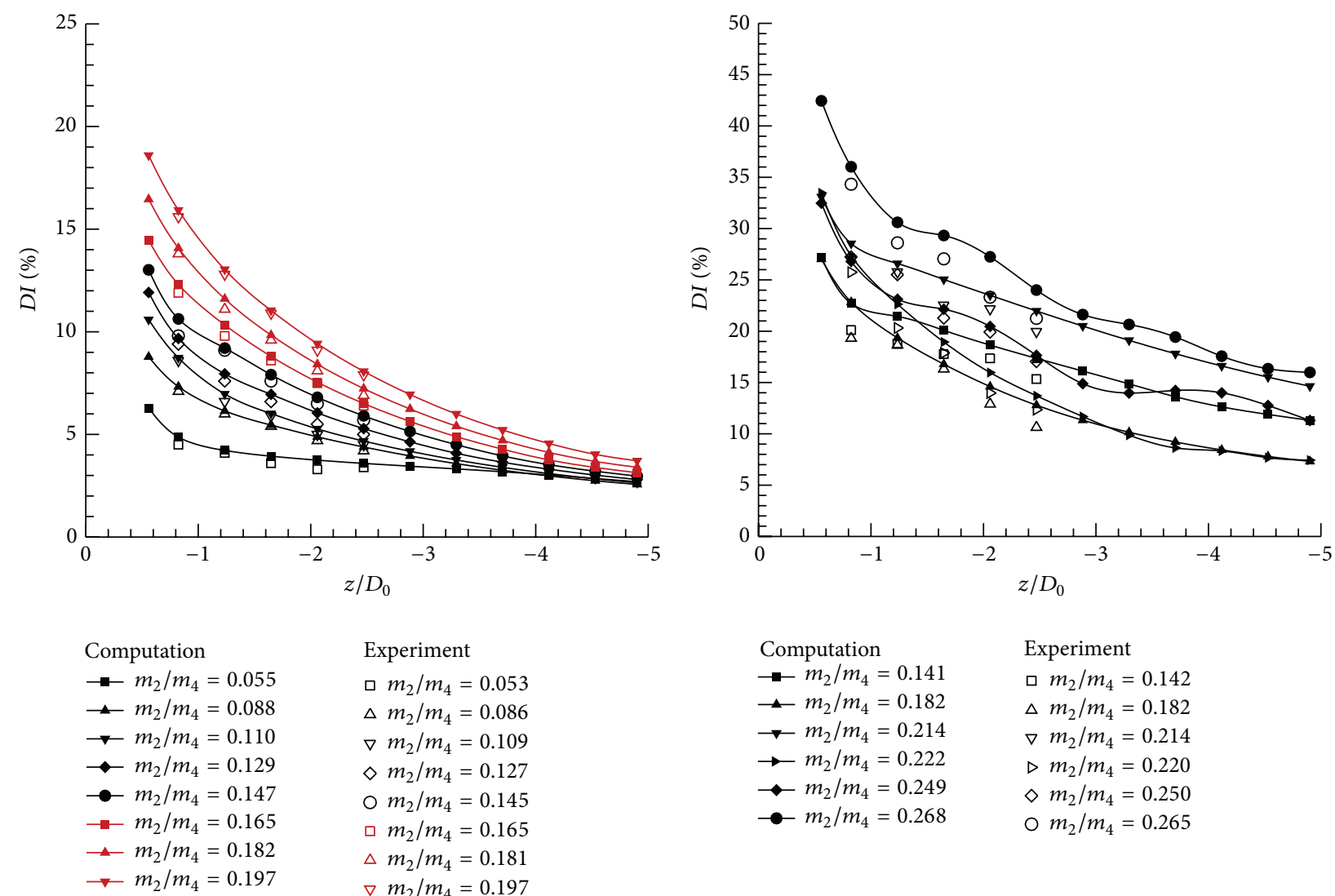

Experiment

$m_{2} / m_{4}=0.053$

$\triangle m_{2} / m_{4}=0.086$

$\nabla m_{2} / m_{4}=0.109$

$\diamond m_{2} / m_{4}=0.127$

$\circ m_{2} / m_{4}=0.145$

ㅁ $m_{2} / m_{4}=0.165$

$\triangle m_{2} / m_{4}=0.181$

$\nabla m_{2} / m_{4}=0.197$

(a)

\begin{tabular}{|c|c|}
\hline $\begin{array}{l}\text { Computation } \\
\rightarrow m_{2} / m_{4}=0.141 \\
\multimap m_{2} / m_{4}=0.182 \\
\longrightarrow m_{2} / m_{4}=0.214 \\
\rightarrow-m_{2} / m_{4}=0.222 \\
\longrightarrow-m_{2} / m_{4}=0.249 \\
\longrightarrow-m_{2} / m_{4}=0.268\end{array}$ & $\begin{array}{l}\text { Experiment } \\
\square m_{2} / m_{4}=0.142 \\
\Delta m_{2} / m_{4}=0.182 \\
\nabla m_{2} / m_{4}=0.214 \\
\triangleright m_{2} / m_{4}=0.220 \\
\diamond m_{2} / m_{4}=0.250 \\
\diamond m_{2} / m_{4}=0.265\end{array}$ \\
\hline
\end{tabular}

(b)

Figure 5: Distortion index $(D I)$ at several locations behind the jet injectors plotted for different values of $m_{2} / m_{4}$. (a) Four jets with equal mass flow rates and (b) four jets with unequal mass flow rates.

develop a methodology to generate a prescribed total pressure distortion pattern. The method is based on the computational procedure for multiple jets in counterflow and the quasi-onedimensional inviscid analysis is used as the starting point to estimate the overall total pressure loss; this method is described in Section 8.

\section{Limits of Operation of the Flow System from Inviscid Analysis}

The methodology developed here to generate a given total pressure distortion pattern is based on the inviscid calculation of the total pressure loss due to a jet issuing into a counterflowing stream. Before the methodology is described it is prudent to investigate the regimes and limits of operation of this flow system in an inviscid framework. The results presented here are for the four jet system with $D_{0}=102 \mathrm{~mm}$ and $d_{j}=7.5 \mathrm{~mm}$. The total mass flow rate from the four jets is used for calculating the total pressure loss because it is uniquely determined by the mass flow ratio $\left(m_{2} / m_{4}\right)$ and the inlet Mach number $\left(M_{1}\right)$. Further, it was seen (in Figure 4) that the results from inviscid calculations are in good agreement with the present experiments and computations. The station numbering is according to Figure 1.

The calculations were conducted for a series of inlet Mach numbers $\left(M_{1}\right)$ ranging from 0.05 to $1\left(\delta M_{1}=0.05\right)$ and various mass flow ratios $\left(m_{2} / m_{4}\right)$. The total pressure loss due to the jets in counterflow is shown in Figure 7(a). Several interesting and important observations can be made from this figure. The $M_{4}=1$ limit is shown at the top of this plot. This limit places a constraint on the meaningful solutions that can be obtained from the inviscid analysis. The $M_{4}=0.6$ limit is also shown in this figure. This limit assumes importance as the mean Mach number at the inlet to the compressor face is usually below 0.6 (Serovy [16], Walsh and Fletcher [17]) to avoid very high relative Mach number at the rotor tip and attendant excessive losses (Saravanamuttoo et al. [18]). The vertical line showing the $m_{2} / m_{4}=0.5$ limit is also depicted in this plot. This is quite a liberal limit as it is not expected to inject more than $50 \%$ of the total mass flow rate from the jets. Another limiting curve is the $p_{02}=p_{01}$ curve; this follows from the fact the flow system under consideration cannot exist if the total pressure in the jet is less than the total pressure in the counterflow stream (Sekundov [19]). As 

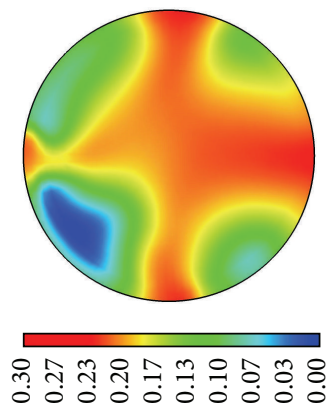

(a)
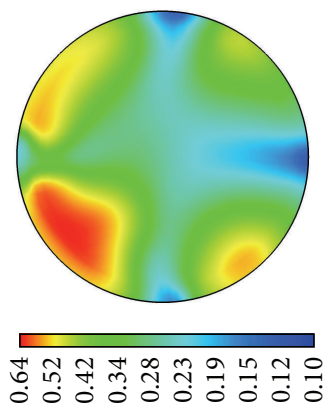

(c)

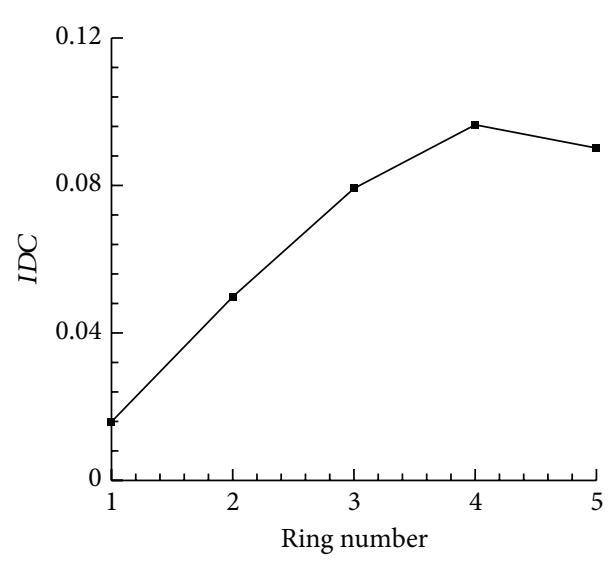

(e)
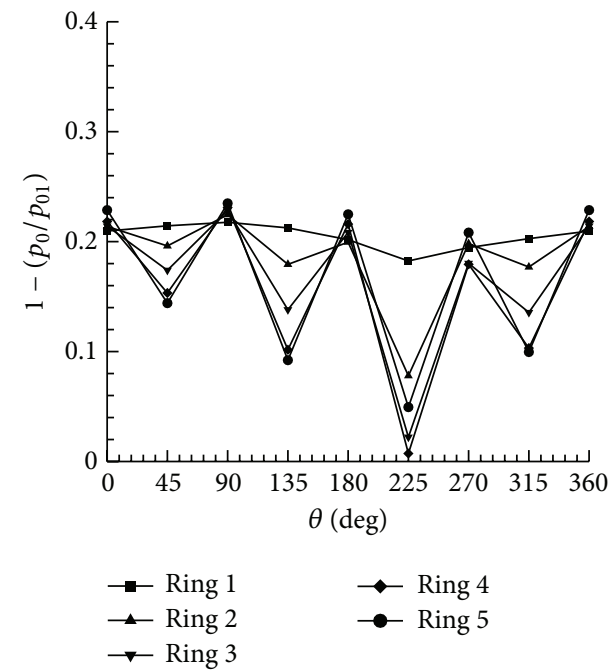

(b)
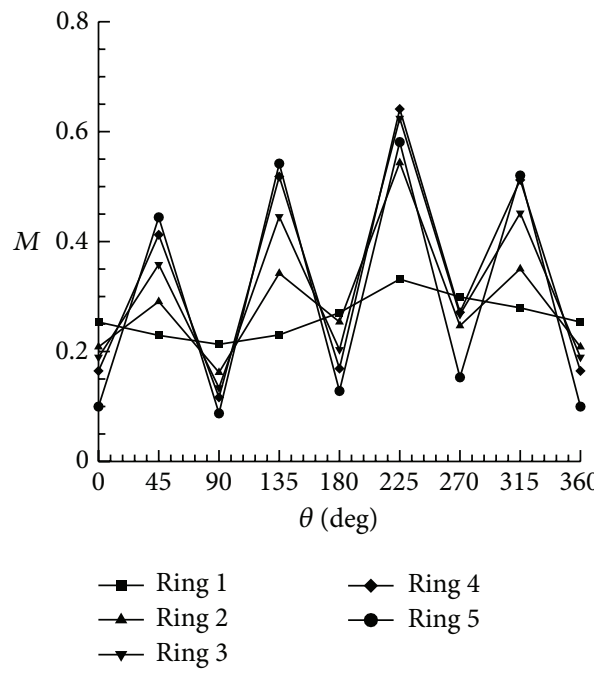

(d)

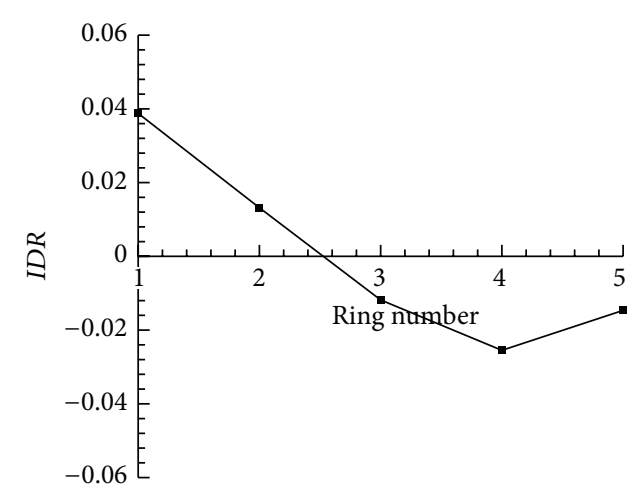

(f)

FiguRE 6: Distortion parameters at the AIP for four jets with unequal mass flow rates $m_{2} / m_{4}=0.214(D I=27.495 \%)$. In this figure (a) contours of total pressure loss $\left[1-\left(p_{0} / p_{01}\right)\right]$, (b) distribution of total pressure loss in each of the five rings, (c) contours of Mach number, (d) Mach number distribution in the five rings, (e) the circumferential distortion parameter IDC, and (f) the radial distortion parameter IDR in the rings. 
$m_{2} / m_{4} \rightarrow 1$, the total pressure loss asymptotically becomes about 0.112 , because, though $m_{1} \rightarrow 0$ in the $m_{2} / m_{4} \rightarrow 1$ limiting process, $p_{01}$ and $p_{02}$ remain finite leading to this asymptotic value.

Now, the exit Mach number $\left(M_{4}\right)$ is plotted in Figure 7(b). In the inviscid analysis the exit velocity at station 4 can correspond to either subsonic or supersonic solution and only the subsonic solution is considered. The results are plotted here for subsonic exit Mach number $M_{4}$ till it becomes unity. All the limits described above are also depicted in this figure. These limits yield the restrictions on the possible values of total pressure loss and exit Mach number and the solution space is shown by a shaded region in the figures.

\section{Methodology}

The methodology to generate a given total pressure distortion pattern is described in this section. The inputs required for this method are the average Mach number $M_{3}$ and the required total pressure distribution at the AIP. (It may be mentioned here that in the inviscid analysis station 3 is not defined. However, in the methodology detailed below the flow properties at station 4 from the inviscid analysis are used at station 3 which is the AIP.)

The procedure to simulate a given total pressure distribution is as follows.

(1) From the given total pressure distribution the average total pressure loss $\left[1-\left(p_{03} / p_{01}\right)\right]_{\text {ave }}$ is calculated. It may be noted that $p_{01}$ is uniform.

(2) The inlet Mach number $\left(M_{1}\right)$ is evaluated from $M_{3}$ and $\left[1-\left(p_{03} / p_{01}\right)\right]_{\text {ave }}$ from Figure 8 .

(3) The inlet mass flow rate $m_{1}$ can be calculated from $M_{1}$ by assuming inlet total pressure $\left(p_{01}\right)$ and total temperature $\left(T_{01}\right)$ using isentropic relations.

(4) The mass flow ratio $\left(m_{2} / m_{4}\right)$ required to exert the required total pressure loss can be estimated from Figure $7(\mathrm{a})$. It has to be ensured that $m_{2} / m_{4}<0.5$ and all the constraints discussed in Section 7 are met.

(5) Now that the overall mass flow ratio $\left(m_{2} / m_{4}\right)$ is known and thus the total mass flow rate $m_{2}$ in the jets, the task at hand is to distribute this mass flow rate amongst the jets. This is done by the following steps.

(a) The flow field is divided into a number of streamtubes equal to the number of jets and each streamtube has one jet issuing into the counterflowing stream.

(b) Since $M_{1}$ which is uniform is known from Step (2) the local mass flow rate in each of the jets $m_{2, j}$ is calculated to effect desired pressure loss in the streamtube. The calculation is similar to the one done for the entire flow field, the only difference being that the calculations are now done for individual streamtubes.

(c) The jets are assumed to be choked and from $m_{2, j}$ and the diameter of the jet $d_{j}$ the jet total pressure is calculated from isentropic relations (see Shapiro [20])

$$
\frac{m_{2, j}}{A_{j}}=0.0404 \frac{p_{02 j}}{\sqrt{T_{02}}} .
$$

(d) At this stage it is ensured that $p_{02 j}>p_{01}$ meeting Sekundov's [19] criterion so that the jet penetrates into the counterflow stream.

(e) A computational simulation is now performed with the parameters obtained from the previous steps. This allows effects of viscosity and turbulence and also the interaction between the jets and assumed streamtubes.

(f) The total pressure distribution at the AIP obtained from computations is compared with the target total pressure distribution.

(g) The difference between the obtained and required total pressure loss is employed to vary the mass flow rates in the jets. The mass flow rate is varied from the current mass flow rate by an amount equal to the difference of the previous and current total pressure loss multiplied by the slope of the $\left[1-\left(p_{03} / p_{01}\right)\right]$ versus $m_{2, j}$ curve for the corresponding inlet Mach number $\left(M_{1}\right)$.

(h) With the new mass flow rate steps (c) to (g) are repeated till convergence.

8.1. Demonstration of the Methodology. The methodology developed to generate a given total pressure distribution was described above in Section 8. In the present section this methodology is demonstrated using an example flow case with the four-jet system. In fact, this flow case was the one for which computations were already done as indicated in Table 1. By using a total pressure distribution which is known a priori and employing the methodology developed here it can be readily asserted whether the scheme can reproduce the known total pressure distribution.

8.1.1. Example 1. Consider the total pressure distribution shown in Figure 9, representing the contours shown later in Figure 10(a). It was obtained from computations (Sl. number 10 in Table 1). This flow case had unequal mass flow rates in the jets thus leading to a characteristic "V" shaped total pressure loss distribution described in Section 6.3; also to be noted is the sharp dip in the total pressure loss distribution at $\theta=225^{\circ}$. Since this problem is designed using the known mass flow rates in the jets it serves as a good test case for the methodology to retrieve the total pressure distribution.

The number of sampling points at that radius was chosen to be eight (equiangularly spaced). It was initially thought that four sampling points can be selected with each sampling point corresponding to each jet. However, a careful look at the total pressure loss distributions reveals that to account for the characteristic peaks and dips a minimum of eight sampling points are required. 


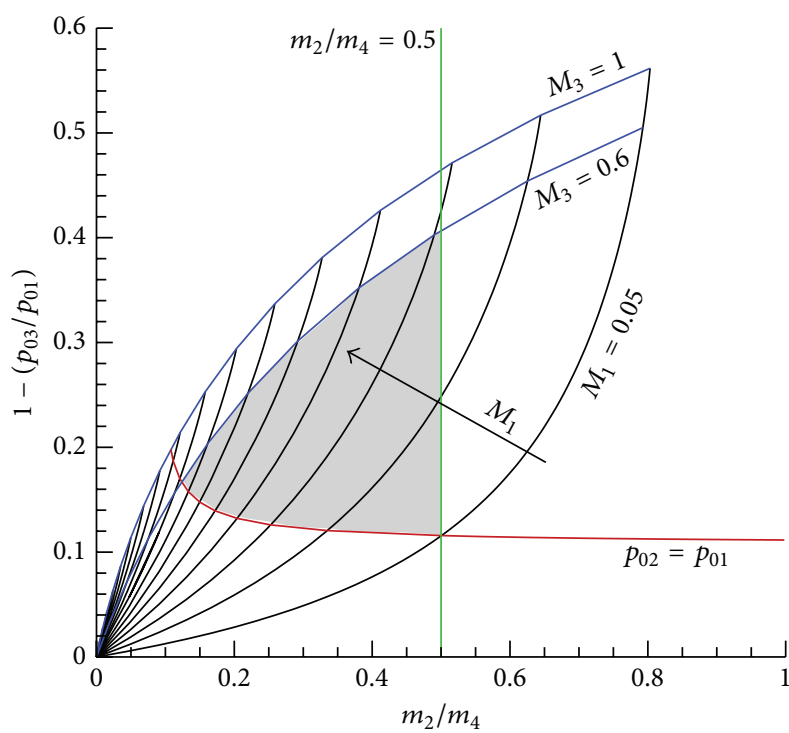

(a)

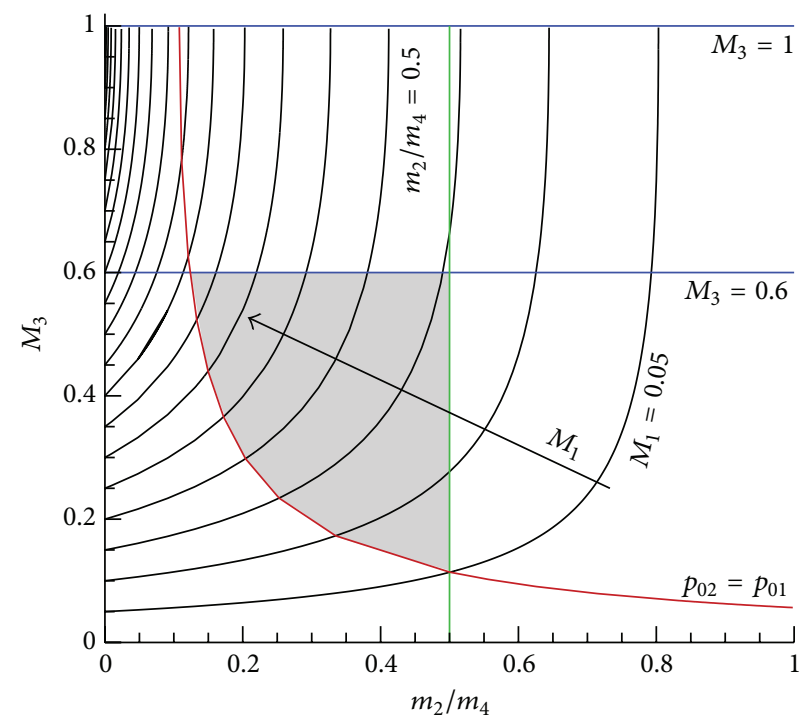

(b)

Figure 7: Variation of (a) total pressure loss and (b) exit Mach number $\left(M_{4}\right)$ as a function of mass flow ratio $m_{2} / m_{4}$ for different values of inlet Mach number $\left(M_{1}\right)$ with $\delta M_{1}=0.05$ obtained by inviscid analysis. The calculations were done for $D_{0}=102 \mathrm{~mm}$, four jets with $d_{j}=$ $7.5 \mathrm{~mm}$.

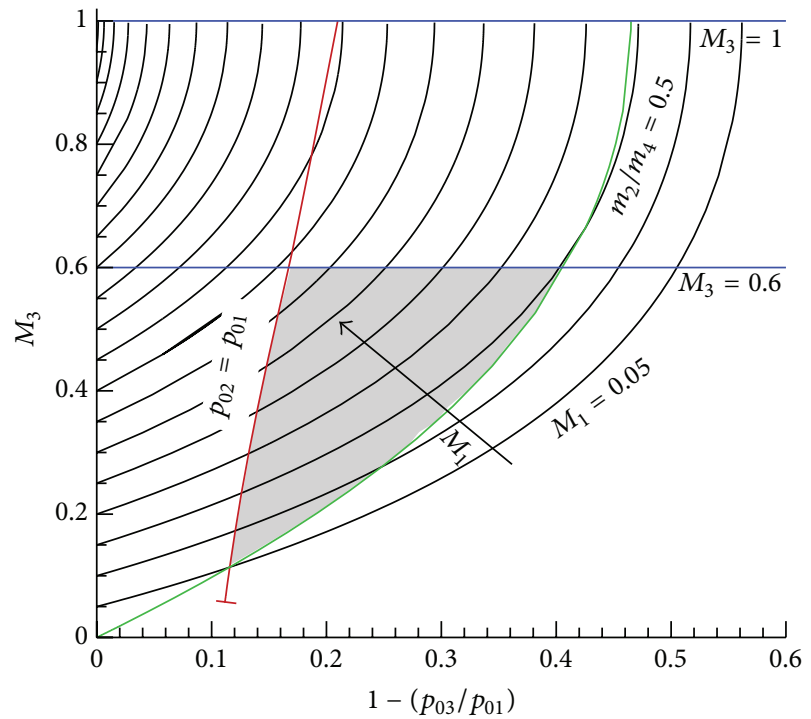

FIgURE 8: Variation of average Mach number $M_{3}$ as a function of total pressure loss for different values of inlet Mach number $\left(M_{1}\right)$ with $\delta M_{1}=0.05$ obtained by inviscid analysis.

The scheme is started with the given total pressure loss distribution in Figure 9 and the required average Mach number at the $A I P, M_{3}=0.315$. From the given total pressure distribution, $\left[1-\left(p_{03} / p_{01}\right)\right]_{\text {ave }}=0.183, M_{1}$ is evaluated to be 0.176 and from these data $m_{2}$ is calculated to be $0.250 \mathrm{~kg} / \mathrm{s}$. This mass flow rate is to be distributed amongst the four jets.

Now, the flow field is divided into four streamtubes with each streamtube having a jet issuing into the counterflow stream. Since eight sampling points are considered and only

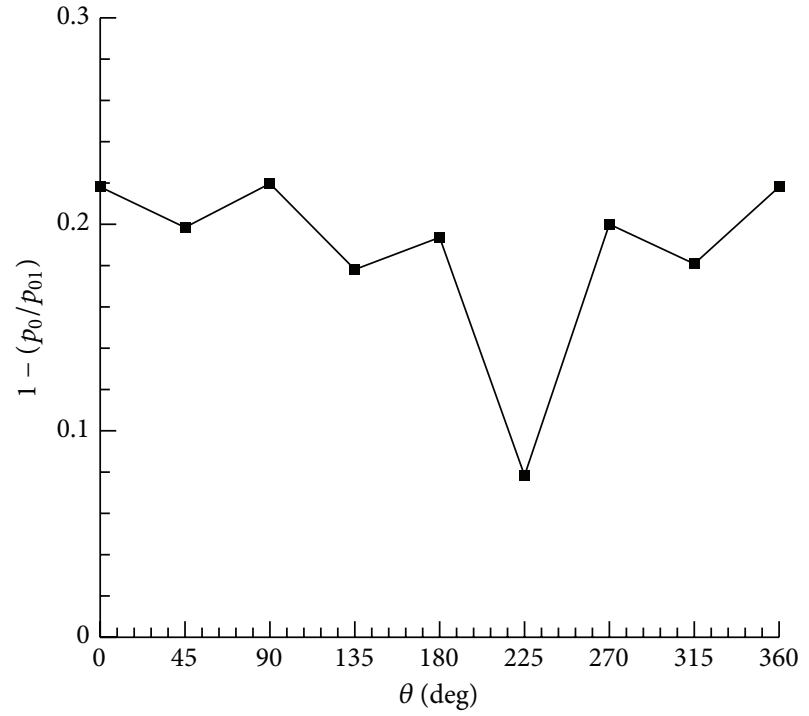

FIgURE 9: Target total pressure loss distribution at the AIP at a radial location $D_{0} / 4$ corresponding to Example 1 .

four jets are available to achieve the desired total pressure loss distribution the total pressure losses are suitably averaged and used to calculate the mass flow rate in the individual jets. The average of three adjacent total pressure losses is used to evaluate the mass flow rate in the jets. Thus the total pressure loss at eight locations can be reduced to four average losses commensurate with the number of jets.

Since $M_{1}$ is known, which is uniform, and from the four average total pressure losses the mass flow rate in each jet can be calculated. At this stage it is not even strictly necessary that 
the mass flow rates from the individual jets sum up to the total jet mass flow rate, though both would be quite close. The total pressure in the jet can be calculated with the jet mass flow rate and the jet diameter assuming a jet exit total temperature.

With the parameters thus obtained a computation is done as per the procedure discussed in Section 3. The total pressure distribution obtained from the computations is compared with the target pressure distribution at all the eight sample points. The difference can be quantified in terms of a rootmean-square error (RMSE) defined as (Hubble and Smith [14])

$$
R M S E=\sqrt{\frac{\sum_{i=1}^{n}\left(p_{0, \text { obtained }} / p_{0, \text { target }}-1\right)^{2}}{n}} \times 100 \%,
$$

where $n=8$ is the number of sampling points.

From these eight total pressure losses and by averaging the adjacent total pressure losses once again the data are reduced back to four loss values. These four values are used to evaluate the new mass flow rates in the jets. The difference between the obtained and target total pressure loss is calculated for each of the four averaged total pressure loss values and this difference is multiplied by the slope of the $\left[1-\left(p_{03} / p_{01}\right)\right]$ versus $m_{2, j}$ curve; the new mass flow rates in the jets are obtained by adding this value to the current mass flow rates. Iteration is continued with these new values till the total pressure distribution obtained from the computations converges to the target distribution. The procedure for the present example converges in ten iterations and the RMSE after the tenth iteration is only $4.443 \%$.

The distortion parameters at the AIP are plotted in Figure 10. The total pressure loss contours at the AIP for the target and those obtained after convergence are shown in Figure 10(a). A good agreement between the patterns is observed. The distortion index for the target solution was $27.779 \%$ and that achieved was $28.019 \%$. The total pressure distribution in the rings normalised by the inlet total pressure is plotted in Figure 10(b); the agreement is good at all the rings. The circumferential and radial distortion elements are plotted in Figures 10(c) and 10(d), respectively; again the agreement is good. The methodology developed here is able to accurately reproduce not only the overall distortion level (in terms of the distortion index) but also the distribution of total pressure in the rings and the individual circumferential and radial distortion components in the rings.

The flow case in this example serves to indicate that a simple minded quasi-one-dimensional inviscid analysis approach used as a starting point is not adequate to generate the target total pressure distribution. Turbulent flow computations are necessary and with a suitable strategy they should iteratively progress towards the target distribution. This test case also gives a hint that close to an exact solution, that is known to exist in this example, there may be approximate solutions and any search methodology, like the present one, may end up with one of them. This is not surprising keeping in mind the evolving nature of the flow field which is governed by equations that are nearly parabolic in nature. In such a system it is possible that two altogether different flow fields but with equal source strengths are likely to evolve into the fully developed or nearly fully developed states that are very close. A methodology trying to search a source strength based on the fully developed state may end up with another source strength distribution which is nearly as good since the final states are close.

It now seems plausible that by increasing the number of jets and consequently the number of sampling points in the system the simulated flow field can get closer to the target pattern.

\section{Simulating Aircraft Distortion Patterns}

In the present section the methodology is demonstrated to generate a more complex given flow field using twenty jets. It may be recalled that in the previous example the target total pressure distortion pattern was known a priori by the computed flow field. In the present section, however, the methodology is deployed for an example whose solution is not known. This example represents distortion pattern typically measured in aircraft inlet/gas turbine engine testing.

The twenty jets are arranged circumferentially at three radii at $0.134 D_{0}, 0.305 D_{0}$, and $0.443 D_{0}$ as shown in Figure 11. The inner diameter of the jets $d_{j}$ was $3.35 \mathrm{~mm}$ and the jets are representative of equal area sectors. The total mass flow rate from the jets is as in (1), but with $N=20$. The timeaveraged computations were performed as in Section 3, with the computational domain consisting of 1,980,440 cells.

Before this example is solved it is pertinent to list some distortion descriptor elements as defined in AIR1419 [11] (see also Williams [21]). These distortion parameters are also relevant apart from the global parameters like distortion index (8) and circumferential and radial distortion components (9) and (10). The rationale for describing such distortion descriptors is well covered in [11]. Briefly, the distortion descriptor must be capable of describing the compressor's stability to the magnitude of circumferentially varying total pressure deficit, the time period a compressor blade spends in the low total pressure region, the number of low total pressure regions encountered by a blade in one revolution, the magnitude of radially varying total pressure defect, and the occurrence of the circumferential and/or the radial total pressure defect in the hub, midspan or tip of the compressor.

The circumferential distortion in each ring is described by the magnitude of circumferentially varying total pressure deficit, the circumferential extent of the low total pressure region and the number of low total pressure regions in terms of intensity, extent, and multiple-per-rev, respectively. These parameters are defined next.

The circumferential extent of the low total pressure region defined as intensity $(\triangle P C / P)$ which indicates the magnitude of total pressure defect in each ring $i$ :

$$
\left(\frac{\Delta P C}{P}\right)_{i}=\frac{\left(p_{0, \text { ring,ave }}\right)_{i}-\left(p_{0, \text { low,ave }}\right)_{i}}{\left(p_{0, \text { ring,ave }}\right)_{i}}
$$

where $p_{0 \text {,ring,ave }}$ is the average total pressure in the ring and $p_{0 \text {,low, ave }}$ is the average of the total pressures below the 


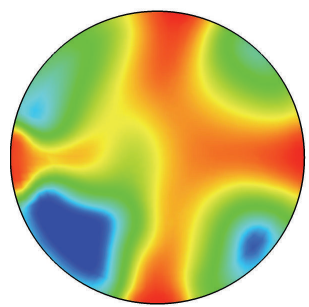

Target

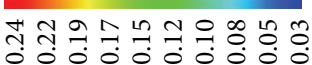

(a)

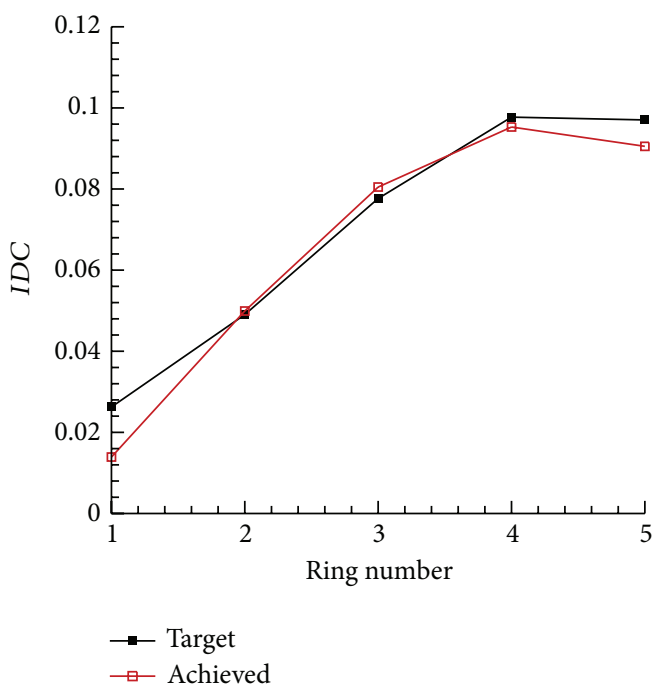

(c)
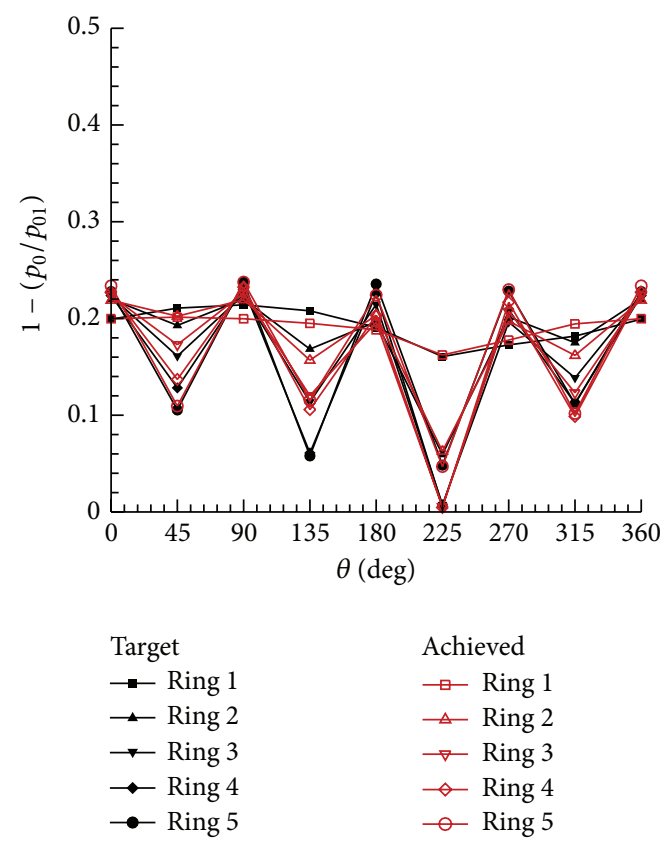

(b)

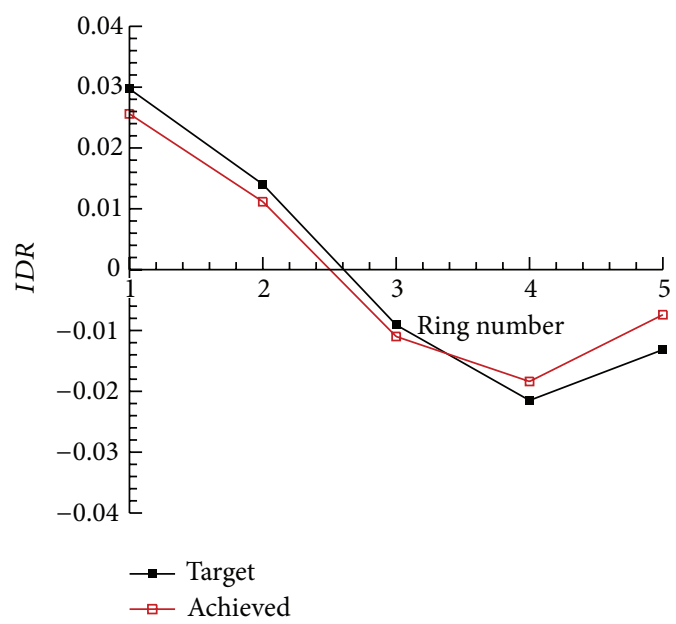

(d)

Figure 10: Distortion parameters at the AIP for Example 1. (a) Total pressure loss contours, (b) total pressure loss distribution in the rings, (c) circumferential distortion parameter IDC, and (d) radial distortion parameter IDR.

ring average total pressure. The counter $i$ indicates the ring number; the number of rings is usually five (see Figure 3). It is worth noting that the circumferential intensity $(\triangle P C / P)$ is akin to $I D C$ with $p_{0 \text {,ring,min }}$ and $p_{0 \text {,face,ave }}$ in the definition of $I D C$ replaced by $p_{0, \text { low, ave }}$ and $p_{0, \text { ave }}$, respectively.

The circumferential distortion extent $\left(\theta^{-}\right)$is the annular extent, in degrees, in which the total pressure is below the average total pressure in the ring.

$$
\left(\theta^{-}\right)_{i}=\theta_{2, i}-\theta_{1, i} .
$$

The number of low total pressure regions in a ring is quantified by multiple-per-rev $(M P R)$ parameter. For total pressure distributions which have $M P R$ more than one $M P R$ can be calculated as ratio of the sum of the areas of the low total pressure regions to the single largest area. For such patterns the circumferential intensity corresponds to the maximum value of the $(\triangle P C / P)$ and the circumferential extent $\left(\theta^{-}\right)$is

$$
\left(\theta^{-}\right)_{i}=\left(\theta_{2, i}-\theta_{1, i}\right)+\left(\theta_{4, i}-\theta_{3, i}\right) .
$$

The radial distortion intensity is the difference between the average total pressure in the plane of interest and the ring 


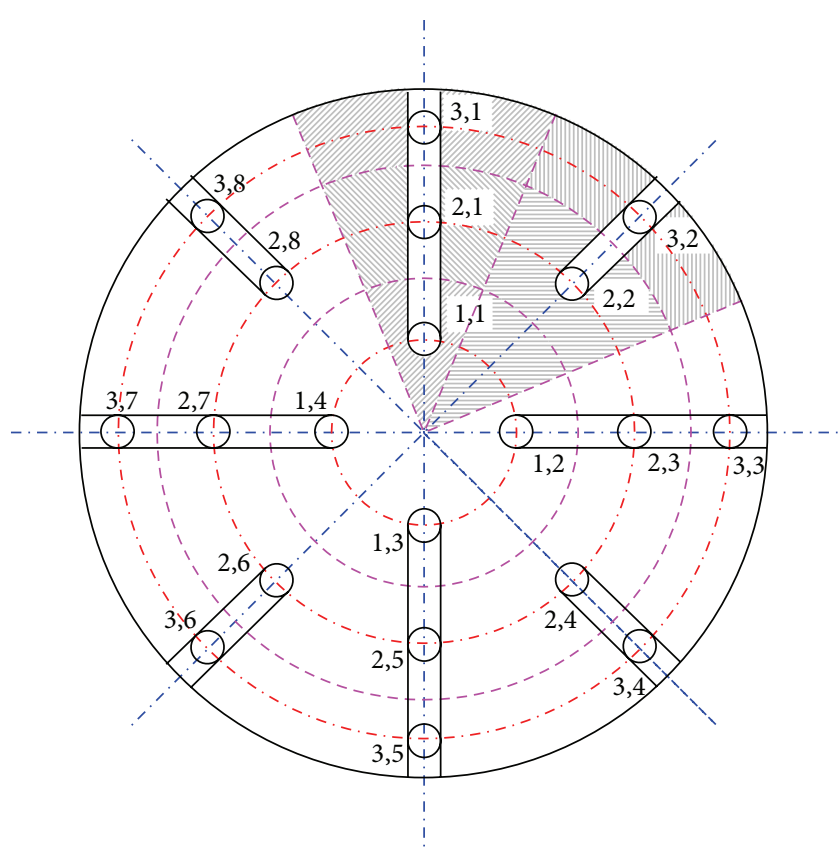

FIGURE 11: Schematic arrangement of the twenty jets in three circumferential locations.

average pressure divided by the average total pressure in the plane. The radial distortion intensity $(\triangle P R / P)$ is defined as

$$
\left(\frac{\Delta P R}{P}\right)_{i}=\frac{p_{0, \text { face, ave }}-p_{0, \text { ring,ave }}}{p_{0, \text { face, ave }}} .
$$

It may be noted that $(\triangle P R / P)$ is identical to IDR defined earlier in (10).

There is a great advantage of using the distortion descriptors defined above. This method avoids the otherwise cumbersome representation of the total pressure distribution by polynomial, spline. or Fourier curve fit and results in consistently good correlations to an acceptable degree of accuracy (see Cousins [22]). With the new distortion descriptors defined, now the flow case example can be considered.

9.1. Example 2. This flow case example is selected from [11, Figure 19]. The target total pressure loss distribution $\left[1-\left(p_{0} / p_{01}\right)\right]$, that is, to be generated at the AIP, is shown in Figure 12(a) (these contours were generated from the data in [11, Figure 19]). This is a $90^{\circ}$ one-per-rev and tip radial combined distortion pattern. It has essentially uniform circumferential and angular extent distortion elements. This pattern was measured from a distortion screen test.

With 40 total pressure data points given it is now only a matter of suitably averaging these pressure data and imposing the right amount of mass flow rate in the jets. With the sampling points obtained after the averaging procedure the methodology to generate the total pressure pattern is initiated and computations are performed. The method converged in three iterations and the RMSE was $3.836 \%$.

The distortion parameters at the AIP after convergence are plotted in Figure 12. It may be mentioned here that we tried to achieve the target total pressure distribution also with four jets and, as an intermediate step, with twelve jets. The obtained total pressure distributions with the jet systems are shown in Figure 12(b). A visual inspection of the total pressure loss contours indicates that with twelve and twenty jets the pattern has similar regions of high and low total pressure regions compared to the target pattern. The ringwise total pressure loss distribution is plotted separately for the five rings for the sake of clarity in Figure 12(c). It was earlier mentioned that the target total pressure pattern was obtained with a screen. The distortion screens have a typical character of inducing sharp total pressure gradients at the edges of the screen from the distorted to undistorted sector (Overall [23]). This sharp transition can be seen in the target distribution in all the rings from about $90^{\circ}$ to about $315^{\circ}$. The jet system is not able to reproduce these steep gradients. The air jet system not capturing the steep gradients was also reported in Braithwaite et al. [24]. In all the rings the total pressure distribution obtained agrees reasonably well with the target distribution.

The circumferential and radial distortion components, $I D C$ and $I D R$, are plotted in Figure 12(d). The IDC values achieved are lower than the target values. The obtained IDR values are higher at the hub and lower near the tip compared with the target values. An inconsistent behaviour of IDC and $I D R$ values was also reported in [19]. The circumferential intensity is lower in all the rings compared to the target values. However, the circumferential extent is captured well. The target pattern had no MPR content; the air jet system had MPR content in the outer rings. The radial intensity is reasonably comparable with the target values. Overall, the air jet system is able to reproduce the target distribution reasonably well in terms of lower RMSE and other distortion parameters. Thus a complex aircraft-type distortion pattern was simulated reasonably well with the air jet system.

\section{Conclusions}

An air jet distortion generation system was developed to simulate the distorted flow field ahead of gas turbine engines in ground test facility. The flow field of four jets in confined counterflow was computationally and experimentally investigated. The total pressure field downstream of the jet injector was obtained for different values of mass flow ratio, $m_{2} / m_{4}$, from 0.055 to 0.197 for equal mass flow rates in the jets and from 0.190 to 0.352 for varying mass flow rates. The total pressure distortion parameters at the Aerodynamic Interface Plane $(A I P)$ were evaluated. This study helped in the use of multiple jets to generate a given target total pressure distribution at the AIP. The following are the conclusions from this study.

(i) The loss in total pressure due to the jets interacting with the counterflow was quantified by a total pressure loss parameter $\lambda_{p 0}$, and this value increased with increasing mass flow ratio.

(ii) The total pressure loss parameter, $\lambda_{p 0}$, was also evaluated by a quasi-one-dimensional inviscid analysis 


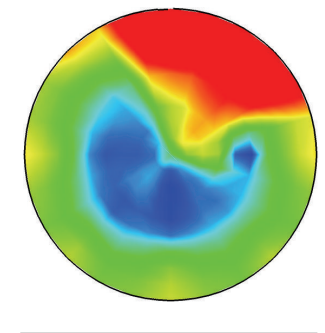

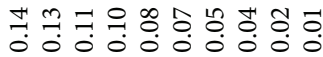

(a) Target
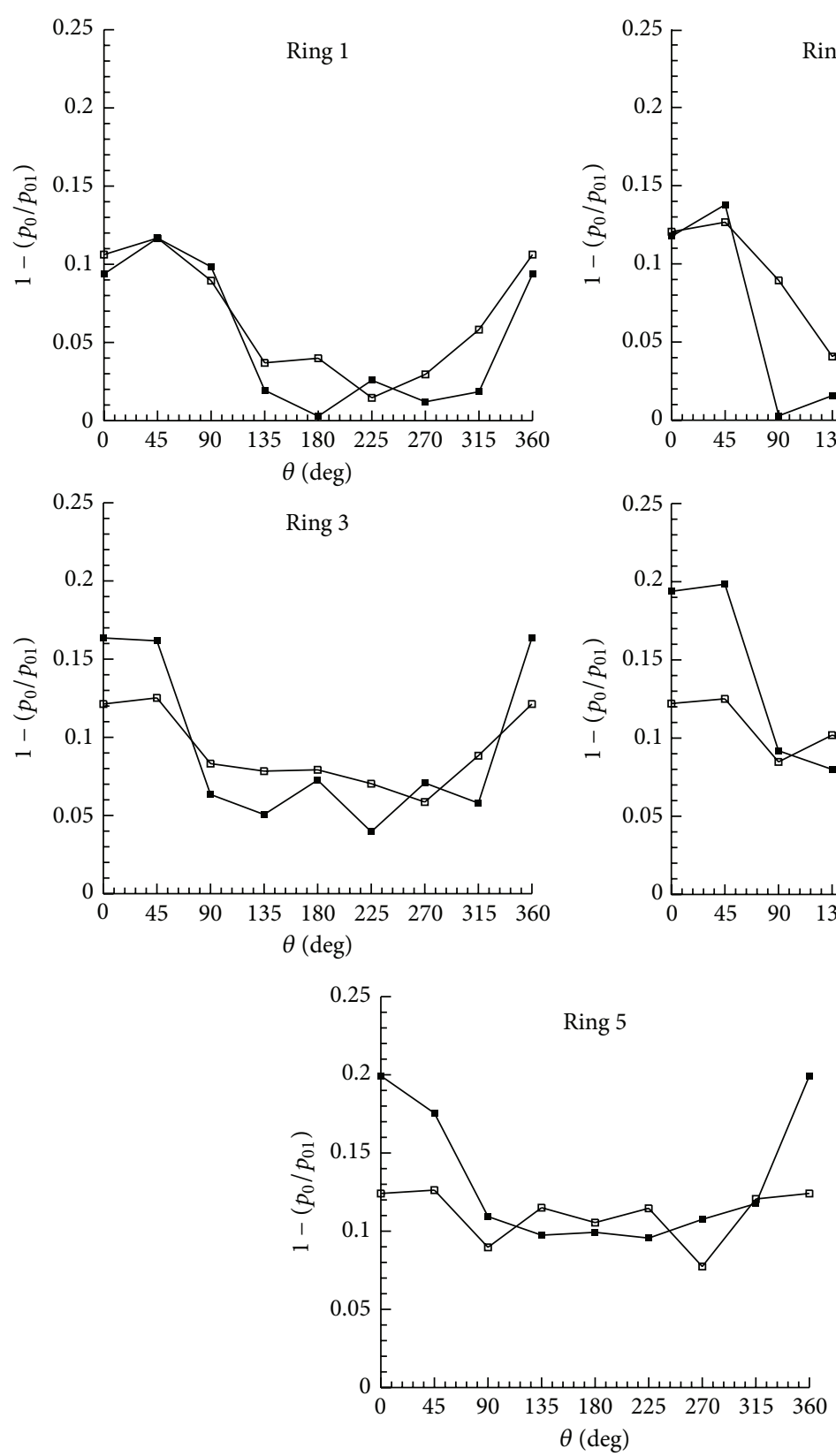

- Target

$\rightarrow$ Achieved

(c)

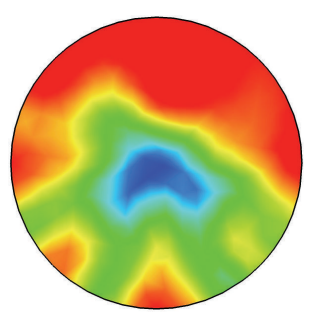

12 jets

(b) Achieved

Ring 5

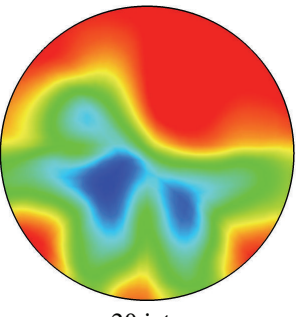

20 jets
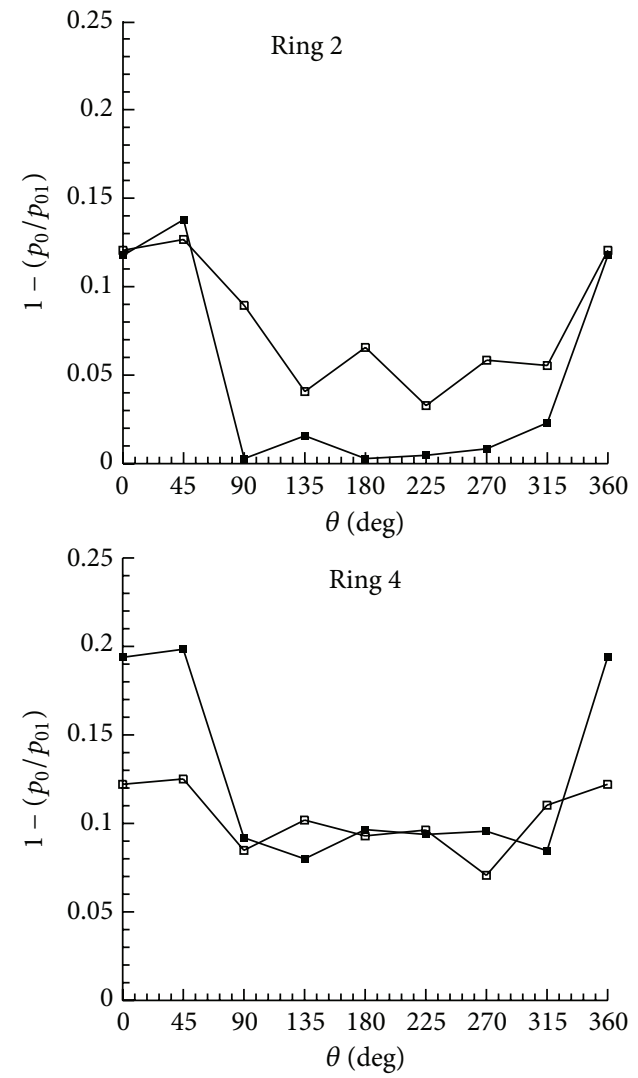

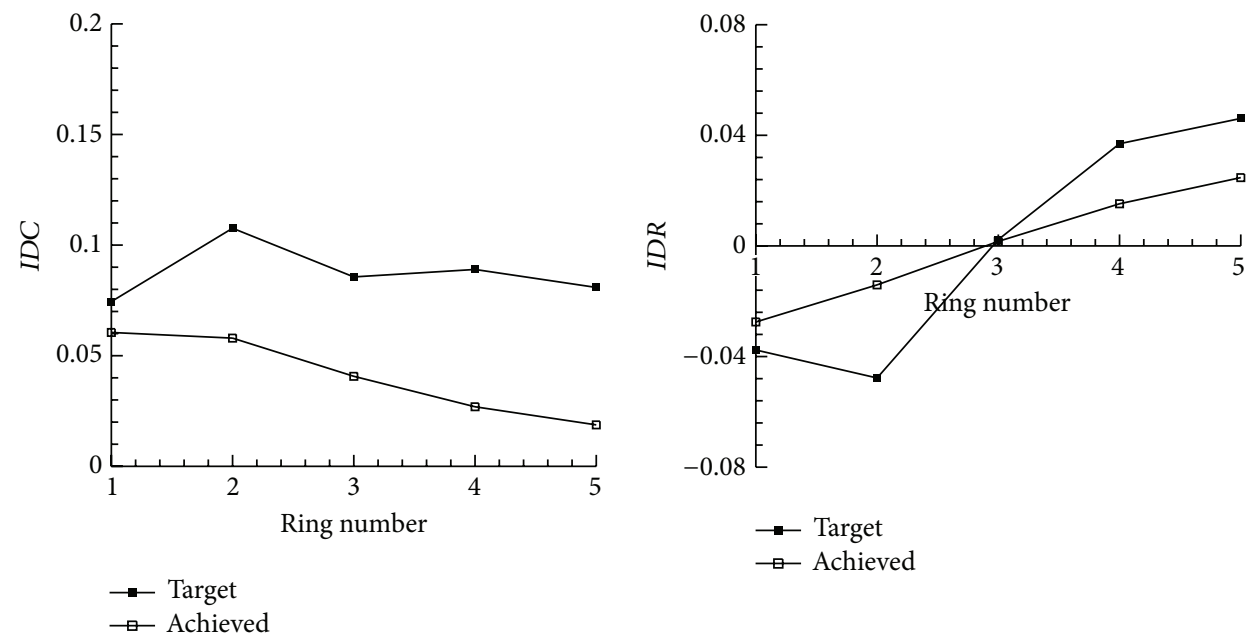

(d)
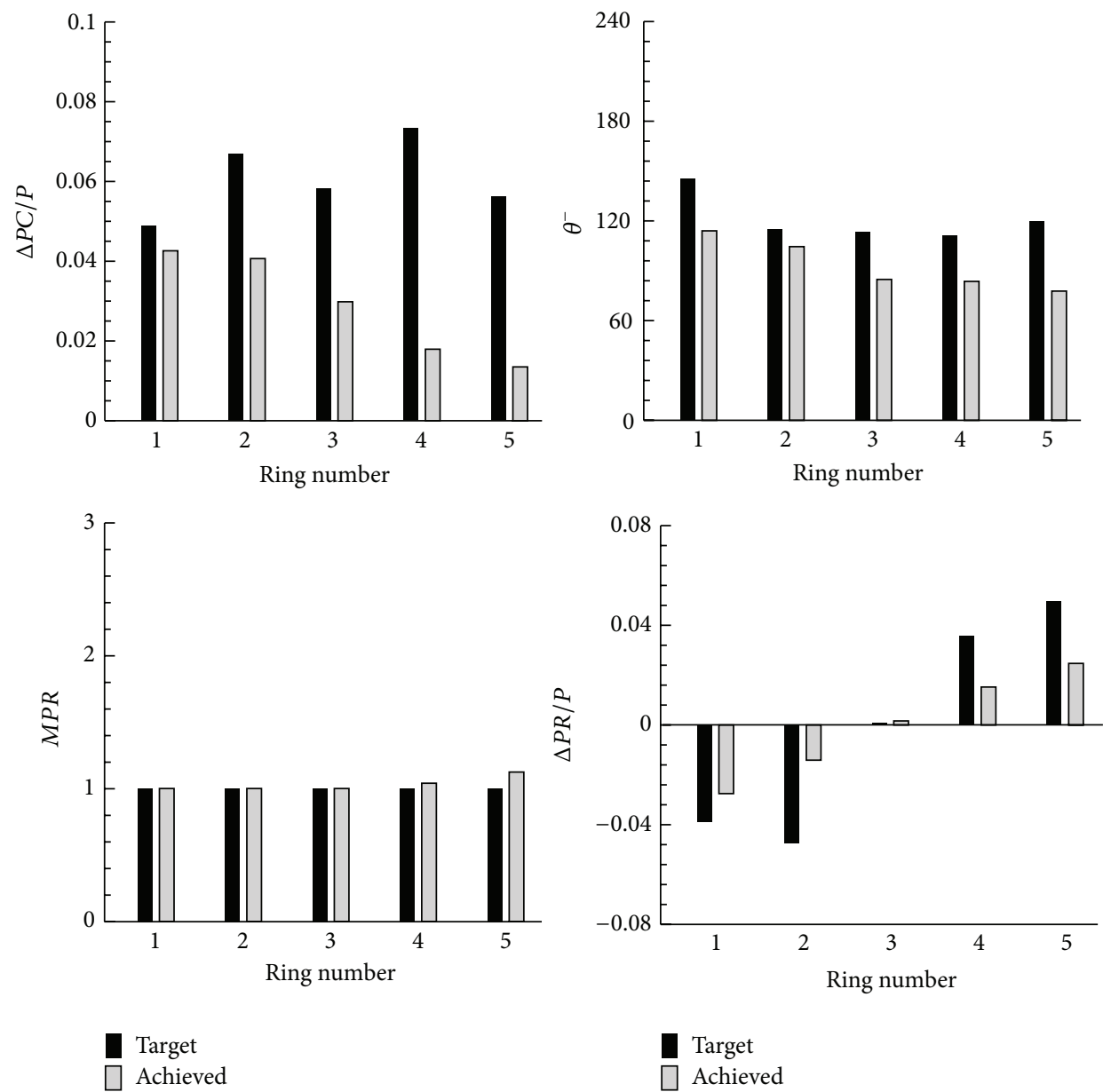

(e)

Figure 12: Contours of (a) target and (b) achieved total pressure loss distributions, (c) ring-wise total pressure loss distribution, (d) circumferential $(I D C)$ and radial $(I D R)$ distortion elements and (e) circumferential distortion intensity $(\triangle P C / P)$, circumferential extent $\left(\theta^{-}\right)$, multiple-per-rev $(M P R)$, and radial distortion intensity $(\triangle P R / P)$.

and was found to be in good agreement with the computations and experiments.

(iii) The total pressure nonuniformity, quantified by distortion index (DI), was found to be highest at a location just downstream of the jet injector and low values of $D I$ were observed at far downstream locations. $D I$ increased with an increase in mass flow ratio at all the planes. DI was found to be high with 
injecting unequal mass flow rates in the jets compared to with equal mass flow rates.

(iv) With the four-jet system, the total pressure loss distribution at the AIP had a characteristic "V" shaped distribution, giving us a hint that to obtain total pressure distributions as required for gas turbine testing the number of jets has to be increased.

(v) With this understanding, a methodology was developed to generate a target total pressure distortion pattern at the AIP. The inviscid analysis helped in identifying the various constraints of the problem and revealed the solution space.

(vi) This methodology was demonstrated for a distortion flow pattern typical of use in gas turbine engine testing using twenty jets, which is a smaller number than reported in the literature. The procedure converged with an RMSE of $3.836 \%$. Apart from reproducing the distortion pattern, the various distortion parameters obtained compared well with the target values.

\section{Nomenclature}

$\begin{array}{ll}(\triangle P C / P): & \text { Circumferential distortion } \\ (\Delta P R / P): & \text { intensity } \\ A_{j}: & \text { Radial distortion intensity } \\ A I P: & \text { Area of the jet nozzle, } \mathrm{m}^{2} \\ C_{1 \varepsilon}, C_{2 \varepsilon}, C_{\mu}, \sigma_{k}, \sigma_{\varepsilon}: & \text { Aerodynamic Interface Plane } \\ & \text { equation } \\ D_{0}: & \text { Diameter of confining duct, } \mathrm{m} \\ d_{j}: & \text { Diameter of jet nozzle, } \mathrm{m} \\ I D C: & \text { Circumferential distortion } \\ I D R: & \text { parameter } \\ k: & \text { Radial distortion parameter } \\ M: & \text { Turbulence kinetic energy, } \mathrm{m}^{2} / \mathrm{s}^{2} \\ m: & \text { Mach number } \\ m_{2} / m_{4}: & \text { Mass flow rate, kg/s } \\ M P R: & \text { Mass flow ratio } \\ N: & \text { Multiple-per-rev parameter } \\ n: & \text { Number of jets } \\ p: & \text { Number of sampling points } \\ R M S E: & \text { Pressure, Pa } \\ \varepsilon: & \text { Root-mean-square error } \\ \theta: & \text { Turbulence kinetic energy } \\ \theta^{-}: & \text {dissipation rate, } \mathrm{m}^{2} / \mathrm{s}^{3} \\ \lambda_{p 0}: & \text { Circumferential location, deg } \\ \mu_{t}: & \text { Circumferential distortion extent, } \\ & \text { deg } \\ & \text { Total pressure loss parameter } \\ & \text { Turbulent viscosity, kg/ms. } \\ & \end{array}$

\section{Conflict of Interests}

The authors declare that there is no conflict of interests regarding the publication of this paper.

\section{References}

[1] J. P. Longley and E. M. Greitzer, "Inlet distortion effects in aircraft propulsion system integration," in Steady and Transient Performance Prediction of Gas Turbine Engines, vol. 6 of Lecture Series LS 183. Advisory Group for Aerospace Research and Development, pp. 1-18, Neuilly sur Seine, Paris, France, 1992.

[2] M. Sivapragasam and S. Ramamurthy, "Inlet flow distortion effects in aircraft gas turbine engine compression systems," Tech. Memo. PR-0901, National Aerospace Laboratories, 2009.

[3] D. K. Beale, K. B. Cramer, and P. S. King, "Development of improved methods for simulating aircraft inlet distortion in turbine engine ground tests," AIAA Paper 2002-3045, 2002.

[4] C. L. Meyer, J. E. McAulay, and T. J. Biesiadny, “Technique for inducing controlled steady-state and dynamic inlet pressure disturbances for jet engine tests," Tech. Memo. TM X-1946, NASA, 1970.

[5] B. W. Overall and R. E. Harper, "The Airjet Distortion Generator System: a new tool for aircraft turbine engine testing," AIAA Paper 77-993, 1977.

[6] A. Naseri, M. Boroomand, and A. M. Tousi, "The effect of inlet flow distortion on performance of a micro-jet engine: part I: development of an inlet simulator," in Proceedings of the ASME International Mechanical Engineering Congress and Exposition (IMECE '12), Paper no. IMECE2012-86865, pp. 317324, Houston, Tex, USA, November 2012.

[7] Society of Automotive Engineers, Gas Turbine Engine Inlet Flow Distortion Guidelines, Aerospace Recommended Practice 1420, 2002.

[8] B. E. Launder and D. B. Spalding, Lectures in Mathematical Models of Turbulence, Academic Press, London, UK, 1972.

[9] S. V. Patankar, Numerical Heat Transfer and Fluid Flow, Hemisphere, New York, NY, USA, 1980.

[10] I. B. Celik, U. Ghia, P. J. Roache, C. J. Freitas, H. Coleman, and P. E. Raad, "Procedure for estimation and reporting of uncertainty due to discretization in CFD applications," Journal of Fluids Engineering, Transactions of the ASME, vol. 130, no. 7, Article ID 078001, 2008.

[11] Society of Automotive Engineers, "Inlet total-pressuredistortion considerations for gas-turbine engines," Aerospace Information Report 1419, 1999.

[12] J. P. Holman, Experimental Methods for Engineers, Tata McGraw-Hill, New Delhi, India, 7th edition, 2001.

[13] J. Seddon and E. J. Goldsmith, Intake Aerodynamics, Blackwell, Oxford, UK, 2nd edition, 1999.

[14] J. D. Hubble and R. E. Smith, "Evaluation of an Airjet Distortion Generator used to produce steady-state, total-pressure distortion at the inlet of a General Electric F101-GE-100 turbofan engine," Final Rept. AEDC-TR-78-73, Arnold Engineering Development Center, Arnold Air Force Base, Tenn, USA, 1979.

[15] R. G. Hercock and D. D. Williams, "Aerodynamic response," in Distortion Induced Engine Stability, Lecture Series LS 72. Advisory Group for A erospace Research \& Development, Neuilly sur Seine, Paris, France, 1974.

[16] G. K. Serovy, "Axial flow compressor aerodynamics," in Aerothermodynamics of Aircraft Engine Components, G. C. Oates, Ed., American Institute of Aeronautics and Astronautics, New York, NY, USA, 1985.

[17] P. P. Walsh and P. Fletcher, Gas Turbine Performance, Blackwell, Oxford, UK, 2nd edition, 2004.

[18] H. I. H. Saravanamuttoo, G. F. C. Rogers, and H. Cohen, Gas Turbine Theory, Pearson, New Delhi, India, 5th edition, 2001. 
[19] A. N. Sekundov, "The propagation of a turbulent jet in an opposing stream," in Turbulent Jets of Air, Plasma and Real Gas, G. N. Abramovich, Ed., pp. 99-109, Consultants Bureau, New York, NY, USA, 1969.

[20] A. H. Shapiro, The Dynamics and Thermodynamics of Compressible Fluid Flow, vol. I, The Ronald Press Company, New York, NY, USA, 1953.

[21] D. D. Williams, "Review of current knowledge on engine response to distorted inflow conditions," in Proceedings of the Engine Response to Distorted Inflow Conditions, Conference Proceedings CP 400, pp. 1-32, Advisory Group for Aerospace Research and Development, Neuilly sur Seine, France, 1987.

[22] W. T. Cousins, "History, philosophy, physics, and future directions of aircraft propulsion system/inlet integration," ASME GT2004-54210, 2004.

[23] B. W. Overall, "A procedure for the design of complex distortion screen patterns for producing specified steady-state total pressure profiles at the inlet of turbine engines," AEDC-TR 7210, Arnold Engineering Development Center, Arnold Air Force Base, Tenn, USA, 1972.

[24] W. M. Braithwaite, J. H. Dicus, and J. E. Moss Jr., "Evaluation with a turbofan engine of air jets as a steady-state inlet flow distortion device," NASA TM X-1955, National Aeronautics and Space Administration, Washington, DC, USA, 1970. 

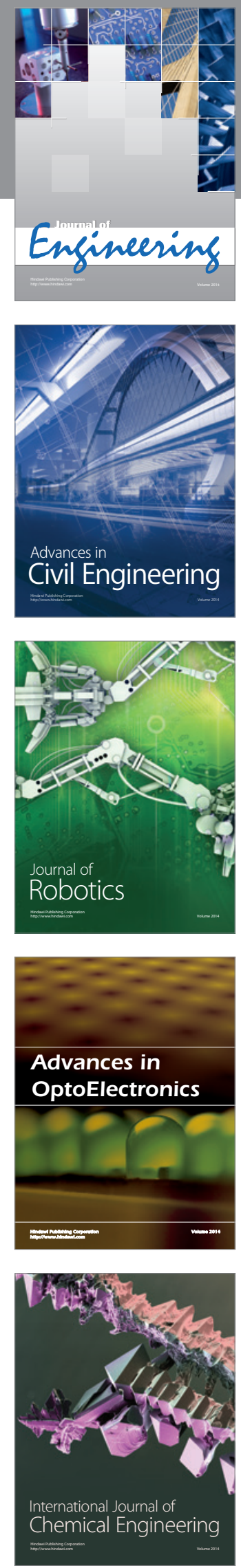

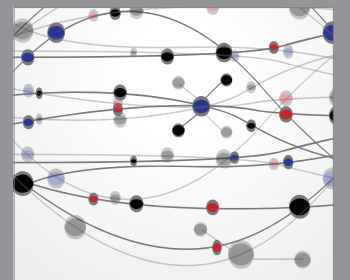

The Scientific World Journal
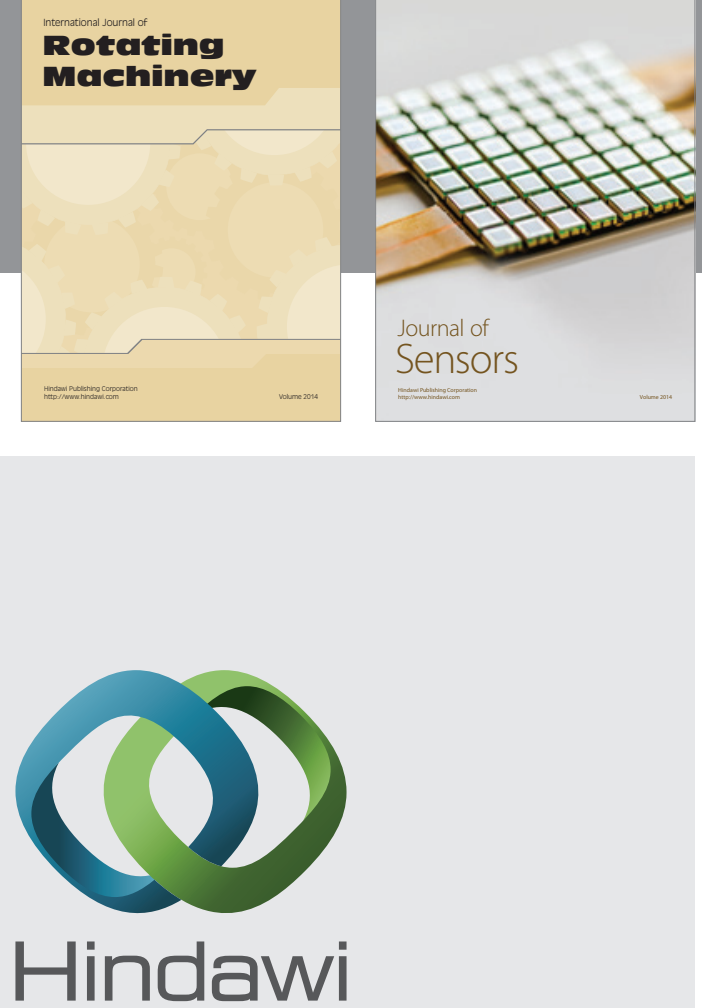

Submit your manuscripts at http://www.hindawi.com
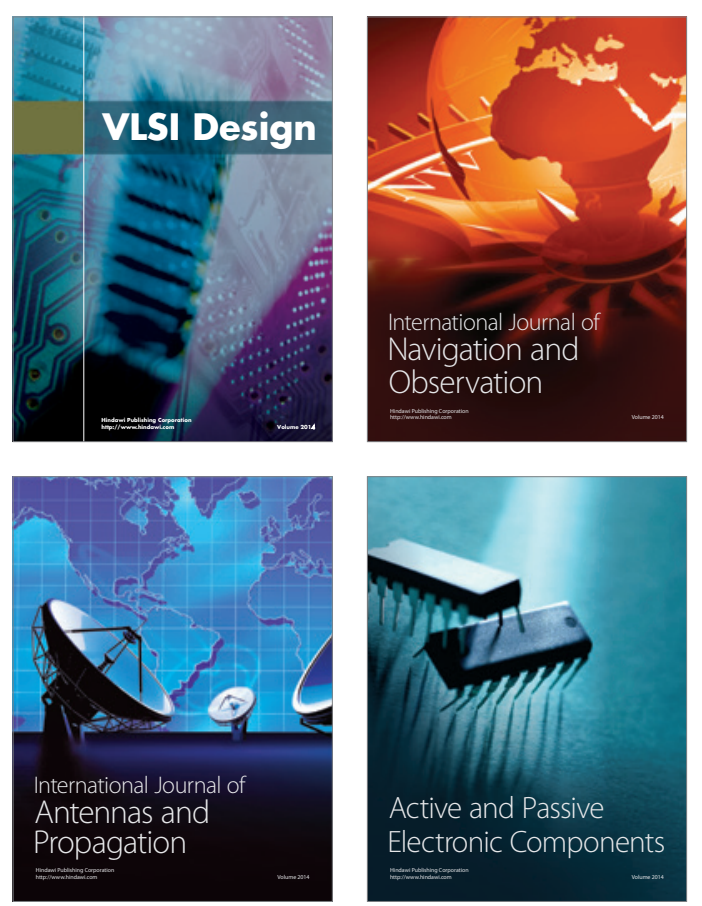
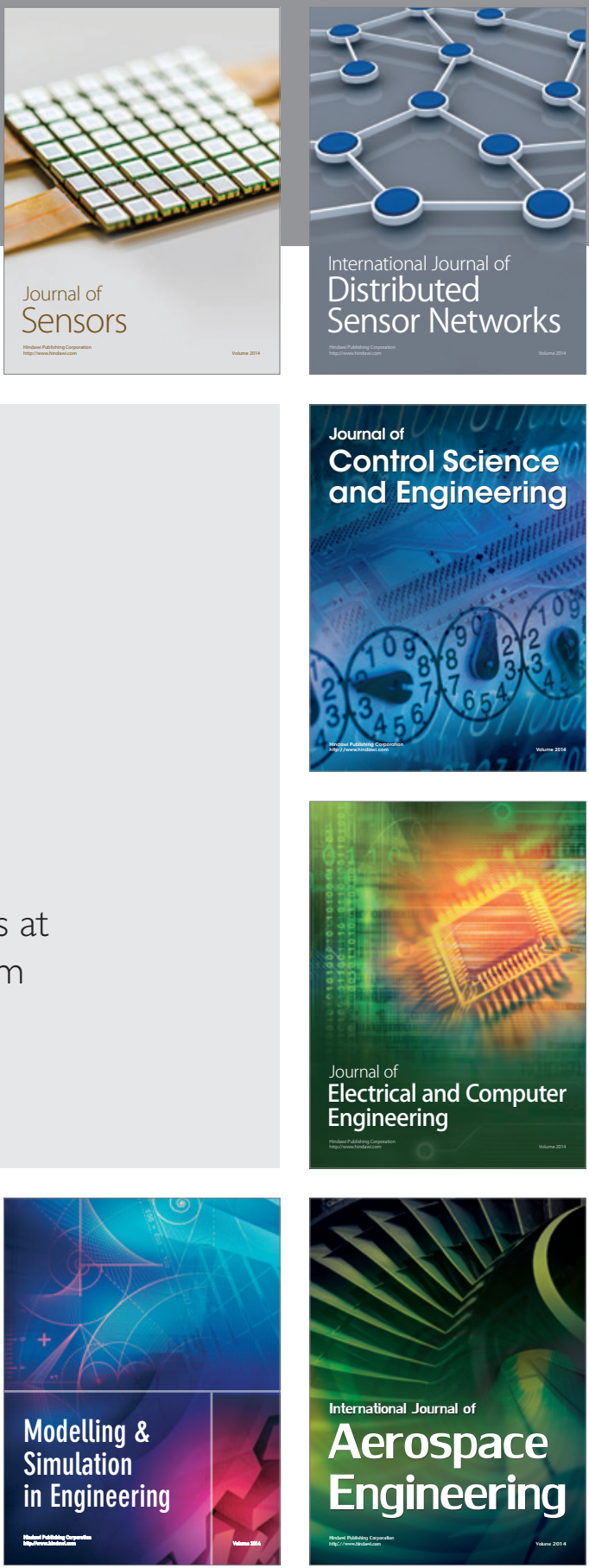

Journal of

Control Science

and Engineering
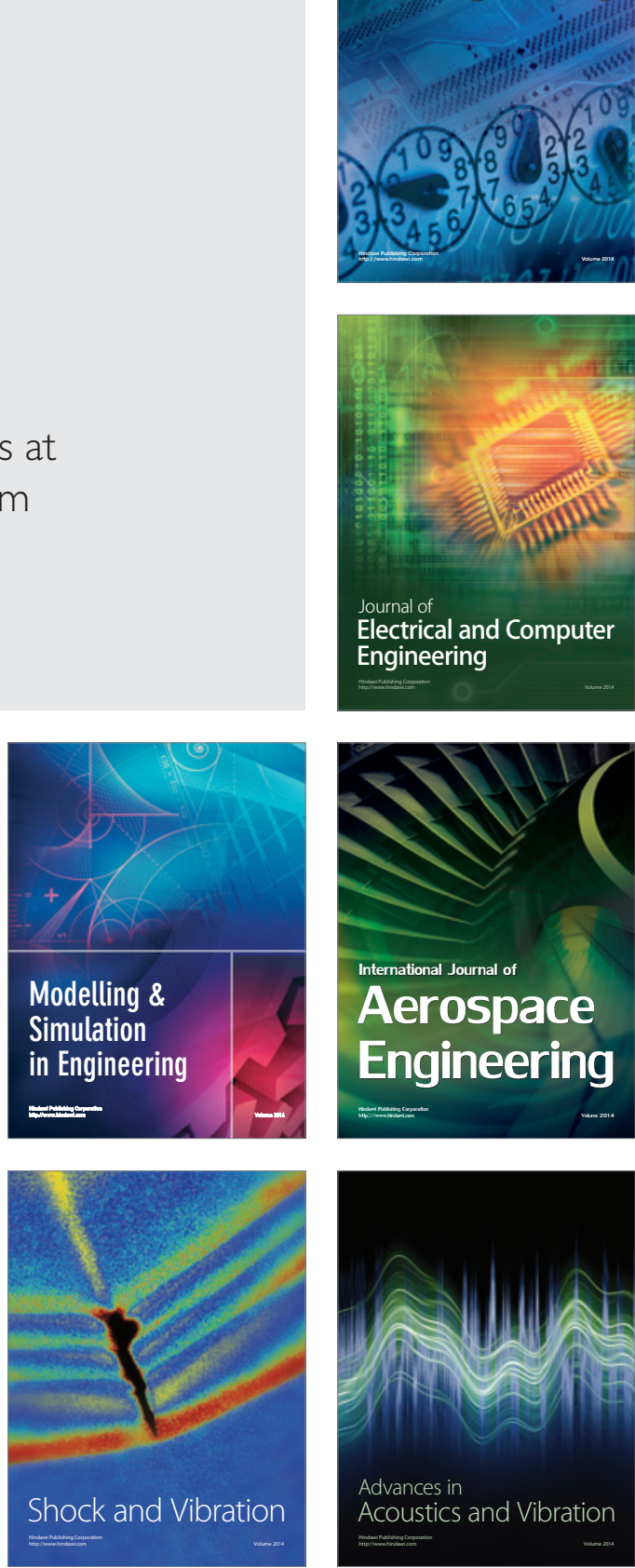